\title{
Functional Recovery and Enhanced Corticofugal Plasticity after Unilateral Pyramidal Tract Lesion and Blockade of Myelin- Associated Neurite Growth Inhibitors in Adult Rats
}

\author{
Werner J. Z'Graggen, Gerlinde A. S. Metz, Gwendolyn L. Kartje, Michaela Thallmair, and Martin E. Schwab \\ Brain Research Institute, University of Zurich and Swiss Federal Institute of Technology Zurich, \\ $\mathrm{CH}-8029$ Zurich, Switzerland
}

\begin{abstract}
After a lesion of the mature CNS, structural plasticity and functional recovery are very limited, in contrast to the developing CNS. The postnatal decrease in plasticity is correlated in time with the formation of myelin. To investigate the possible role of an important myelin-associated neurite growth inhibitor (NI-250; IN-1 antigen), one pyramidal tract of adult Lewis rats was lesioned (pyramidotomy), and the rats were treated with the antibody IN-1, a control antibody, or no antibody. Functional recovery was studied from postoperative day 14 until day 42 using a food pellet reaching task, rope climbing, and a grid walk paradigm. The corticofugal projections to the red nucleus and
\end{abstract}

Functional and anatomical repair of the injured adult CNS is very limited (for review, see Donoghue, 1995, 1997; Schwab and Bartholdi, 1996). In contrast, neuroanatomical plasticity, or the restructuring of neural connections in response to lesions of the CNS, is a well documented phenomenon in the neonatal age group. After unilateral neonatal pyramidotomy in rodents, corticoefferent fibers from the same side as the lesion were found to cross the midline to form new connections with medullary nuclei and to descend to spinal cord levels (Kalil and Reh, 1982). Evidence that new neural connections occur after perinatal brain damage in children is supported by several clinical studies (Farmer et al., 1991; Carr et al., 1993; Cao et al., 1994). Structural neuroplasticity is thought to play an essential role in recovery of function, because animals sustaining CNS lesions at a young age are known to recover much better than those sustaining similar lesions at maturity (Kennard, 1936, 1938; Whishaw and Kolb, 1988; Armand and Kably, 1993).

The lack of large scale remodeling after adult CNS lesions is

\footnotetext{
Received Dec. 29, 1997; revised March 4, 1998; accepted March 26, 1998.

This work was supported by grants of the Swiss National Science Foundation (Grants 31-45549.95 and 4038-043918); the Biotechnology Program of the European Union, Brussels; the Dr. Eric Slack-Gyr-Foundation, Zurich; the American Paralysis Association, Springfield, NJ; the International Spinal Research Trust, Guildford, Surrey, England; the International Research Institute for Paraplegia, Zurich; and the Binelli-Ehrsam-Foundation, Zurich. We thank Professors P. Streit, M.-C. HeppReymond, and C. E. Bandtlow for their help, and Drs. A. McKinney, K. Fouad, J. Tönnes, and C. Wenk for valuable discussions. We also thank R. Schöb for photographic support; E. Hochreutener for graphical support; Drs. R. Dürr, H. J. Kasper, and R. Kägi for technical support; B. Niederöst for cell cultures; M. Weber, R. Schneider, H. Frei, and E. Gubler for help with the histology; and S. Kaufmann for secretary work.

W.Z. and G.M. contributed equally to this paper.

Correspondence should be addressed to Gerlinde Metz, Brain Research Institute, August Forel-Strasse 1, CH-8029 Zürich, Switzerland.

Dr. Kartje's present address: Neurology Service, Edward Hines Jr. Veterans Affairs Hospital, Hines, IL, 60141, and Departments of Neurology and Cell Biology, Neurobiology and Anatomy, Loyola University, Maywood, IL, 60153.

Copyright (C) 1998 Society for Neuroscience $\quad 0270-6474 / 98 / 184744-14 \$ 05.00 / 0$
}

basilar pontine nuclei were analyzed after survival times of 2 and 16 weeks.

Treatment with the monoclonal antibody $I \mathrm{~N}-1$ resulted in almost complete restoration of skilled forelimb use, whereas all the control groups showed severe and chronic impairments. This functional recovery was paralleled by sprouting of the corticorubral and the corticopontine fibers across the midline, thus establishing a bilateral, anatomically specific projection.

Key words: structural plasticity; rat reaching; motor function; motor system; nucleus ruber; pons; corticospinal tract; injury

not well understood, but may be attributable to several reasons, including a limitation of adult neuronal growth potential, a lack or decrease in trophic factors or guidance molecules, or the presence of growth inhibitory molecules. In this regard, limits on the capacity for mature CNS plasticity may be similar to those recently identified for CNS regeneration, for which inhibitory signals present on CNS myelin have been shown to play a crucial role (for review, see Schwab and Bartholdi, 1996). These specific proteins (NI-35 and NI-250) induce long-lasting growth cone collapse and inhibition of neurite growth in vitro (Caroni and Schwab, 1988a; Bandtlow et al., 1990). Neutralization by the specific monoclonal antibody (mAb) IN-1 allowed neurites to grow over myelin or cultured oligodendrocytes (Caroni and Schwab, 1988b). In vivo experiments in rats resulted in longdistance regeneration of lesioned corticospinal tract (CST) fibers (Schnell and Schwab, 1990, 1993; Schnell et al., 1994) and partial recovery of locomotor function after spinal cord injury (Bregman et al., 1995).

The role of myelin inhibitory factors in structural plasticity of the CNS is less clear. In experimentally induced myelin-free spinal cord segments, dorsal root fibers sprouted into vacated territory after sectioning neighboring roots (Schwegler et al., 1995), and after unilateral pyramidotomy the remaining CST sprouted across the spinal cord midline in young adult rats (Vanek et al., 1998).

We questioned whether mAb IN-1 application can enhance plasticity of intact projections and functional recovery after unilateral pyramidotomy. Rats were tested in a skilled forelimb reaching task, which is known to depend on an intact CST (Castro, 1972; Whishaw et al., 1993). Rope climbing and grid walk were used to measure grip strength and limb coordination.

Our behavioral results demonstrate a very high degree of functional recovery in all of these tasks. The anatomical studies revealed a new bilateral innervation to the nucleus ruber and the 
basilar pontine nuclei by intact corticof ugal fibers after blockade of the myelin-associated neurite growth inhibitors with the mAb IN-1. These results reflect a degree of functional recovery and anatomical plasticity after adult CNS lesion that has been previously observed only after perinatal lesions.

\section{MATERIALS AND METHODS}

Sixty-two adult Lewis rats of either sex ranging in age from 45 to $120 \mathrm{~d}$ and in weight from 160 to $430 \mathrm{gm}$ were used. The animals were divided into the following groups: (1) lesion and no antibody treatment $[n=16$; pyramidotomy (PT) only]; (2) lesion and IN-1 antibody treatment $(n=$ $16 ; \mathrm{PT}+\mathrm{mAb}$ IN-1); 3. lesion and control antibody treatment $(n=16 ; \mathrm{PT}$ + anti-HRP); (4) no lesion and no antibody treatment ( $n=6$; anatomy); (5) sham lesion and IN-1 antibody treatment ( $n=4$; mAb IN-1 only); and (6) sham lesion and control antibody treatment $(n=4$; anti-HRP only).

In 24 animals the corticofugal anatomy was analyzed $14 \mathrm{~d}$ after lesion. The other 38 animals were part of a behavioral study and survived for 16 weeks. For statistical purposes the sham-lesioned either mAb IN-1- or control antibody-treated animals were not distinguished during behavioral testing and were analyzed as a single group (sham-operated, antibody only). The experimental animals were identified by code numbers, and investigators were blind to the treatment groups. All animal experiments were performed under supervision of the cantonal veterinary department of Zurich.

\section{Pyramidotomy}

A unilateral pyramidotomy at the level of the caudal medulla oblongata was performed in 48 animals (Fig. 1; groups 1, 2, and 3) to lesion selectively the fibers of the CST (Kalil and Reh, 1982; Vanek et al., 1998). Briefly, animals were pretreated with atropine $(0.025 \mathrm{mg}$, i.p.; Sintetica S.A., Mendrisio, Switzerland) and anesthetized with ketamine $[100 \mathrm{mg} / \mathrm{kg}$ body weight, i.p. (Ketalar, Parke-Davis); additional doses of ketamine were given $(10 \mathrm{mg}$, i.m.) whenever necessary, depending on the reflex status of the animal] and fentanyl $(0.002 \mathrm{mg} / \mathrm{kg}$, i.p.; Hypnorm, Janssen, Buckinghamshire, England). The animals were placed in the supine position, and we used a ventral approach to expose either the right (animals that survived $14 \mathrm{~d}$ ) or the left (animals that were part of the behavioral study) medullary pyramid by opening the bone overlying the pyramidal tract. The dura was opened, and the pyramid was cut $1.5 \mathrm{~mm}$ rostral to the decussation with a sharpened no. 11 scalpel blade. The lesion was covered with gelfoam, and the wound was sutured close. After surgery, all animals received midazolam $(0.1 \mathrm{mg} / \mathrm{kg}$, i.p.; Dormicum, Roche, Basle, Switzerland) and were kept warm on a heating plate until they were fully awake. Eight animals (groups 5 and 6; sham-operated) underwent the same surgical procedure, including the incision of the dura, except that no lesion was performed.

In 12 animals (groups 1,2 and 3; four animals out of each group) that underwent behavioral testing, a second lesion of the left medullary pyramid was performed 2 weeks after the last testing period. Therefore the same surgical procedure was used as described above, with the difference being that the left pyramid was re-exposed and cut $1 \mathrm{~mm}$ rostral to the previous lesion.

\section{Antibody application}

Antibody-secreting hybridoma cells were raised by immunization of mice with NI-250 (Caroni and Schwab, 1988b). These mouse hybridoma cells produce an Ig M antibody against the rat neurite growth inhibitory proteins NI 35/250. Monoclonal control antibodies against horseradish peroxidase (anti-HRP, antibody without inhibitory effect on HRP enzymatic activity) were generated from the same parent myeloma line (P3U; Schnell and Schwab, 1990). To achieve a constant antibody supply, living hybridoma cells were implanted into the CNS. Before implantation, hybridoma cells were tested for inhibitor neutralizing activity in a bioassay using neurons or fibroblasts on a myelin-protein substrate (Rubin et al., 1995) and were also tested for Ig M production by FITC-coupled anti-mouse antibodies. A cell suspension $(6 \mu \mathrm{l})$ containing a total of $10^{5}$ cells was injected stereotaxically with a Hamilton syringe into the hippocampus of the side opposite the lesion (coordinates: $4 \mathrm{~mm}$ caudal, 5 $\mathrm{mm}$ lateral to bregma, and $5 \mathrm{~mm}$ deep). This location was chosen to avoid damage to motor systems by the injection or growth of the cells and to be as close as possible to the ventricular system.

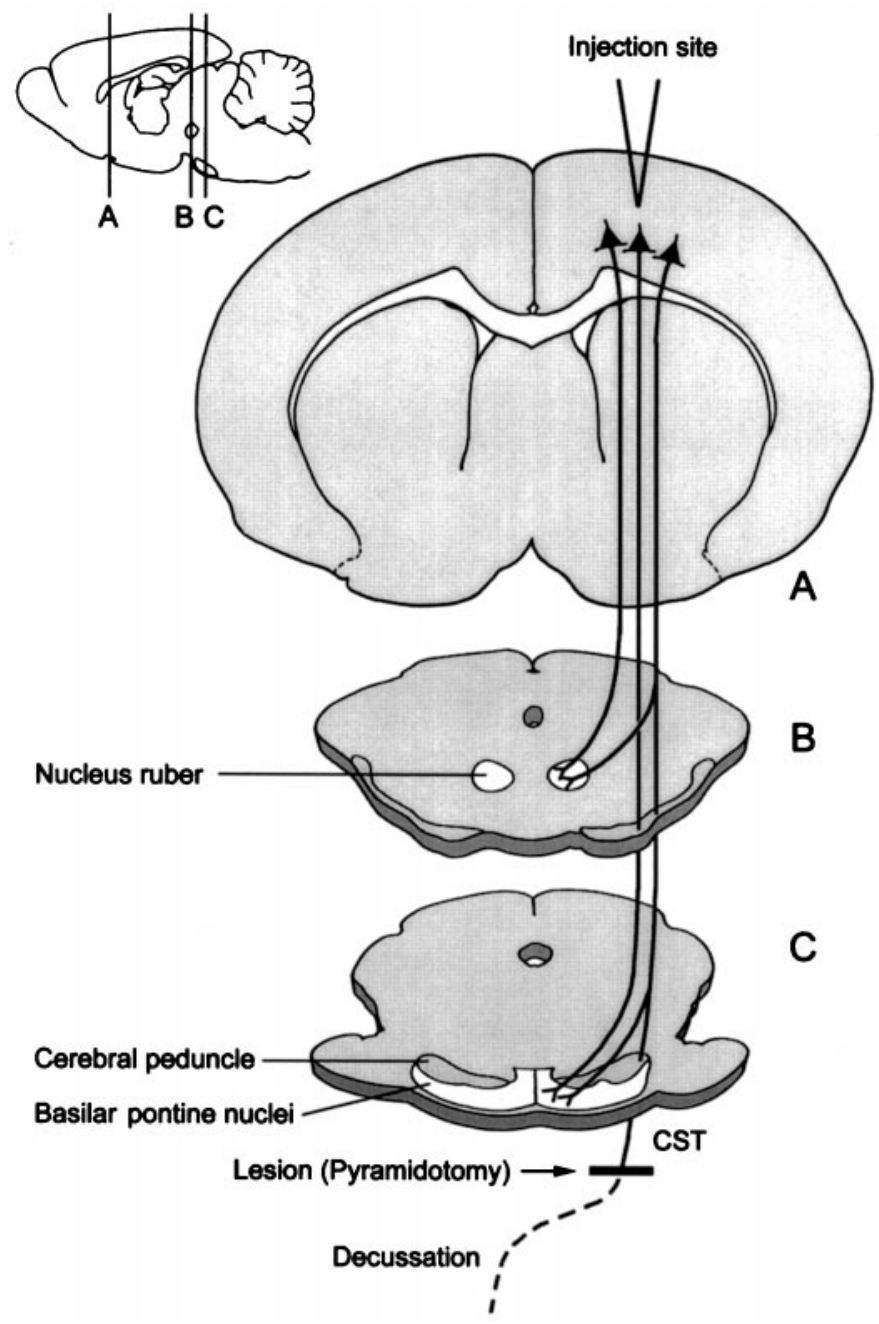

Figure 1. Scheme of the corticof ugal projections from the primary motor cortex to the nucleus ruber, to the basilar pontine nuclei, and to the spinal cord with the cortical BDA tracer injection site and the lesion site (arrow). The insert in the top left corner indicates the levels of the cross sections.

\section{Medical treatment}

One day before pyramidotomy, all animals received an injection of cyclosporin A (10 mg/kg, i.p.; Sandimmun, Novartis, Basle, Switzerland), and an antibiotic regimen was begun with Co-trimoxazol $(0.83 \mathrm{ml} / \mathrm{kg}$, i.p.; Bactrim, Roche). Animals received daily cyclosporin A injections and twice-a-day antibiotic injections for $8 \mathrm{~d}$ (animals that underwent behavioral testing) or $14 \mathrm{~d}$ (other animals). Using cyclosporin $\mathrm{A}$ as an immunosuppressant, the hybridoma xenograft was not rejected, and delivery of antibody was ensured. All animal groups received exactly the same medical treatment.

\section{Behavioral procedures}

During the behavioral testing period, the animals were housed in cages of three to five individuals. A $12 \mathrm{hr}$ light/dark cycle was maintained, starting at 7 A.M. During the entire experimental period the animals received water ad libitum. Before testing, the animals were reduced to $\sim 90 \%$ of their original body weight by maintaining them on a restricted diet for all testing days.

All animals were trained up to 3 weeks preoperatively, and final baseline measurements were recorded. The results of the last session of the preoperative testing were defined as baseline measurements. After completion of preoperative testing, the rats underwent unilateral pyramidal tract section or sham operation and, depending on the experimental group, antibody treatment. To allow for postoperative recovery and because of essential daily cyclosporine A and antibiotic treatment, the postoperative testing sessions started 2 weeks after surgery. Testing 
sessions were performed once daily at the same time of day, $5 \mathrm{~d}$ a week for 4 weeks in the food pellet reaching, rope climbing, and grid walk paradigms with an interval of $1 \mathrm{hr}$ resting time between each task. Video recordings ( 25 frames/sec) for all tests were made in weekly intervals on days $15,21,28,35$, and 42 postoperatively.

For animals that underwent a second lesion of the pyramidal tract, the last testing session (postoperative day 42 after first lesion) was taken as new baseline measurement. These animals were also allowed to recover for 2 weeks after the second lesion before they were retested for 2 weeks.

Food pellet reaching task. This paradigm was performed as described by Kartje-Tillotson and Castro (1980), using a transparent Plexiglas chamber $(30 \times 36 \times 30 \mathrm{~cm})$ with a rectangular opening $(1.5 \times 3 \mathrm{~cm})$ in the front wall on the left side. A smooth Plexiglas shelf was attached outside underneath the rectangular opening. Small round food pellets (45 mg; Bilaney Consultants, Frenchtown, NJ) were placed one after the other onto the shelf, at a distance of $1.5 \mathrm{~cm}$ from the opening. A plastic bar between the shelf and the opening prevented scooping of pellets. Thus, animals were forced to grip and carry the pellets into the testing chamber. The position of the opening on the left side biased animals to use the right, i.e., impaired forelimb.

During preoperative training, rats were placed in the testing chamber for 30 min a day until they learned to reach through the opening for food pellets and grasp and eat them. In the 20-pellet paradigm, which was performed daily, the animal had to obtain 20 pellets, which were stabilized on the smooth surface of the shelf. The parameters that were measured included (1) the amount of time it took to obtain all 20 pellets, (2) the success rate, i.e., the number of pellets grasped and placed into the mouth, and (3) the maximal number of attempts to obtain one pellet. An attempt was recorded if the rat extended its forelimb through the opening. If animals used the ipsilateral limb for reaching or did not start to reach at all, a maximum time of 5 min was given before the session was finished.

Qualitative measurements from video recordings were obtained as subjective disability scores, as modified from Whishaw et al. (1993). The parameters that were analyzed were (1) aim, (2) advance, (3) digits open, (4) pronation, (5) grasp, (6) supination, (7) food release, (8) movement initiation, and (9) movement stop.

Each of these movements was rated on a four-point scale: 0 for normal movements in $>95 \%$ of the observations; 1 for movements that appeared slightly abnormal in $<50 \%$ of the observations; 2 for abnormal movements in $>50 \%$ of the observations; or 3 for no movements or if other parts of the body compensated for movements.

Rope climbing. This test served to examine the grip strength of both forelimbs and hindlimbs and the coordination between the limbs. The ability of the animals to climb a 160-cm-long vertical rope of $4 \mathrm{~cm}$ diameter to reach a platform (Carlini et al., 1967) was tested. The number of foot slips of the affected forelimb and hindlimb was counted (total number of foot slips/total number of steps). The time to climb the rope was recorded to standardize the climbing speed. No reinforcement was given. This test was performed only until postoperative day 21 , because error rates increased with body weight.

Grid walk. Coordination between forelimbs and hindlimbs and accurate limb placement were examined by assessing the ability to cross a 1-m-long runway of metal grid bars with randomly assigned gaps, changing from session to session and ranging from 1 to $5 \mathrm{~cm}$ (modified from Kunkel-Bagden et al., 1993). The performance of each animal was analyzed by counting the number of errors in foot placement for the impaired and the unimpaired side (total number of errors/total number of steps). The time to cross the grid was also recorded. No reinforcement was given.

Statistical analysis. Analysis of behavioral data was performed with a StatView 4.53 statistical package (Abacus Concepts, Berkeley, CA). For comparison of the means within one session, a one-way ANOVA was used for parametric data (time measurements), and a Kruskal-Wallis test was used for nonparametric data (number of errors and attempts, success rates). Differences were investigated further using Scheffe's test or a Wilcoxon signed rank test, respectively. For the latter, a $p$ value $<0.05 /$ number of samples (Bonferroni correction) was chosen as a significance level.

For comparison between the same experimental units, i.e., between baseline and postoperative testing, a paired $t$ test for parametric data and a Wilcoxon signed rank test for nonparametric data were used. All data are presented as means $\pm \mathrm{SEM}$.

\section{Tracing}

In all animals the caudal forelimb area of the primary motor cortex (Neafsey et al., 1986) of the hemisphere corresponding to the lesioned pyramidal tract was traced with the anterograde tracer biotin dextran amine (BDA) (10,000 molecular weight; Molecular Probes, Eugene, $\mathrm{OR})$. In the group of animals that survived $14 \mathrm{~d}$, this area was identified by intracortical microstimulation, and BDA was applied iontophoretically just before the pyramidotomy. The animals that were used for behavioral testing were traced after the last testing period. These animals were pressure-injected with BDA into the caudal forelimb area.

Identification of forelimb primary motor cortex using intracortical microstimulation. Animals were anesthetized with ketamine and secured in a stereotaxic frame, and a right craniotomy was made to expose the sensorimotor cortex. The dura was covered with mineral oil, and to prevent cortical swelling the cisterna magna was cannulated. Five lowthreshold points in the previously described caudal forelimb area (Neafsey et al., 1986; Rouiller et al., 1993) of the primary motor cortex were identified using low-impedance tungsten microelectrodes. Stimulation was applied with a train duration of $60 \mathrm{msec}(0.2 \mathrm{msec}$ pulses and 2.8 msec delay) at a depth of 1.5-2.0 mm. In a systematic grid-like pattern, the motor cortex was mapped, and evoked movements were visually recorded according to type of movement, laterality, depth of best response, and threshold (i.e., lowest possible current that evoked a visible movement). With this paradigm, current thresholds were primarily below $12 \mu \mathrm{A}$ (lowest thresholds were $6 \mu \mathrm{A}$ ). To prevent cortical damage, the applied current was never higher than $25 \mu \mathrm{A}$.

Iontophoretic tracing. To precisely label the projections from the forelimb motor cortex, BDA was delivered iontophoretically (Graybiel and Devor, 1974). Micropipettes with a tip diameter of $20 \mu \mathrm{m}$ were filled with a $10 \%$ BDA solution in $0.01 \mathrm{M}$ phosphate buffer, $\mathrm{pH}$ 7.2. In each animal the five points that elicited the lowest threshold forelimb responses were injected stereotaxically at the exact depth defined by intracortical microstimulation. Positive current of $5 \mu \mathrm{A}$ was applied ( $7 \mathrm{sec}$ pulses every 14 $\mathrm{sec}$ ) over $15 \mathrm{~min}$ using a constant current source. After this procedure the dura was covered by gelfoam, the skull was closed with dental cement, and the skin was sutured.

Tracing by pressure injection. Animals were secured in a stereotaxic frame, and a left craniotomy was made to expose the cortex ipsilateral to the pyramidal lesion. A $5 \mu \mathrm{l}$ Hamilton syringe fitted with a glass micropipette with an opening diameter of $50 \mu \mathrm{m}$ was filled with a $10 \%$ BDA solution in $0.01 \mathrm{M}$ phosphate buffer, $\mathrm{pH}$ 7.2. A single injection of $0.5 \mu \mathrm{l}$ BDA solution was made into the area corresponding to the forelimb according to the earlier experiments $(0.5 \mathrm{~mm}$ rostral and $2.5 \mathrm{~mm}$ lateral from bregma at a depth of $1.5 \mathrm{~mm}$ ). After this procedure the dura was also covered by gelfoam, the skull was closed with dental cement, and the skin was sutured.

\section{$B D A$ histochemistry}

After a survival period of 2 weeks after injection of BDA, all animals were deeply anesthetized with pentobarbital $(450 \mathrm{mg} / \mathrm{kg}$, i.p.; Nembutal, Abbott Laboratories, Cham, Switzerland) and perfused transcardially with Ringer's solution containing 100,000 IU/1 heparin (Liquemin, Roche) and $0.25 \% \mathrm{NaNO}_{2}$ followed by the fixative (4\% paraformaldehyde in $0.1 \mathrm{~m}$ phosphate buffer with 5\% sucrose). The brains and upper spinal cords were removed, post-fixed overnight, and then transferred to a $30 \%$ sucrose solution for $3 \mathrm{~d}$. The tissue was embedded in a gelatinchicken albumin solution polymerized with $25 \%$ glutaraldehyde and immediately frozen by immersion (in $-40^{\circ} \mathrm{C}$ cold isopentane); $50-\mu \mathrm{m}$ thick sections were cut on a freezing microtome. The sections were collected in a solution of $50 \mathrm{~mm}$ Tris-buffered $0.9 \%$ saline, $\mathrm{pH} 8.0$, with $0.5 \%$ Triton X-100 (TBST-X), and then serially mounted on superfrost slides (Menzel-Gläser) according to the semi-free-floating technique of Herzog and Brösamle (1997). Slides were washed for 30 min three times in TBST-X before incubation overnight with an avidin-biotin-peroxidase complex diluted in TBST-X (ABC elite; Vector Labs, Burlingame, $\mathrm{CA}$ ) according to the instructions of the manufacturer. The following day the slides were washed again and preincubated for $10 \mathrm{~min}$ with $0.4 \%$ ammonium nickel sulfate (Sigma, St. Louis, MO), followed by a second preincubation with $0.4 \%$ ammonium nickel sulfate and $0.015 \% 3.3^{\prime}$ diaminobenzidine (DAB) (Sigma, Buchs, Switzerland). The tissue was then reacted in $0.4 \%$ ammonium nickel sulfate, $0.015 \% \mathrm{DAB}$, and $0.004 \% \mathrm{H}_{2} \mathrm{O}_{2}$ in $50 \mathrm{~mm}$ Tris buffer, $\mathrm{pH}$. The process was stopped by washing. The sections were air-dried, lightly counterstained with cresyl violet, and coverslipped with Eukitt (Kindler, Freiburg, Germany). The 
presence of the antibody-producing hybridoma xenografts was checked macroscopically.

\section{Neuroanatomical analysis}

The corticofugal projections to the ipsilateral and contralateral nucleus ruber, separated for the parvocellular and magnocellular parts, and to the ipsilateral and contralateral basilar pontine nuclei were analyzed quantitatively. For all analyses the slides were coded and the investigator was blinded as to the treatment group under analysis. All of the brain areas that were studied were identified with the atlas of Paxinos and Watson (1986). In each animal the lesion site was examined on coronal or longitudinal sections of the medullary pyramid. In some animals the BDA injection sites in the cortex were also examined on cross sections of the primary motor cortex for location, depth, and area of tracer spread.

Quantification of CST labeling. To take into consideration the possible interanimal differences in BDA tracing, the number of labeled fibers in the cerebral peduncle ipsilateral to the injection site was estimated for each animal. The same midpontine level was chosen for each animal, and two consecutive sections per animal were analyzed. Electronic images were acquired with a Xillix Microimager slow-scan, high-resolution CCD camera attached to a Zeiss axiophot microscope. First, the area of the cerebral peduncle was measured by using a $10 \times$ objective and the MCID-Programe (M2-Analyzing Programe, Imaging Research, Ontario, Canada). Then a square of $2975.5 \mu \mathrm{m}^{2}$ was placed four times in a systematic way over the area of the peduncle, and the BDA-positive fibers within these squares were counted at a magnification of $320 \times$. This area corresponded to $\sim 3 \%$ of the total cerebral peduncle cross section. The four values were averaged, and the total number of BDA-positive fibers was extrapolated for each section. The values obtained from the two consecutive sections were averaged.

Quantification of corticorubral and corticopontine projection. The corticofugal innervation from the primary forelimb motor cortex to the nucleus ruber and the pons contralateral to the injection site was analyzed by counting all BDA-positive fibers crossing the midline on each section. To correct for the interanimal differences in section number comprising the red nucleus and the pons, the analyzed total number of sections studied for each animal was randomly reduced to that of the animal with the smallest number of sections. To correct for the differences in the tracing, the number of the BDA-positive, midline-crossing fibers was divided by the total number of labeled CST fibers (calculated as described above). The experimental groups were compared statistically using the ANOVA test.

The density of innervation of the ipsilateral and contralateral basilar pontine nuclei was determined densitometrically. We used a Xillix Microimager to acquire electronic images at a rostral, middle, and caudal level of the pons. Densitometric measurements were performed with the MCID-Programe (M2-Analyzing Programe, Imaging Research Inc.) by outlining the labeled areas of the contralateral and ipsilateral sides. A background correction was performed (unlabeled, neighboring areas), and a ratio of ipsilateral versus contralateral side was calculated in percent. In addition, the densitometric values obtained from the ipsilateral side, normalized by division through the amount of BDA-positive fibers to eliminate variations in tracing, was calculated. Statistical significance was assessed with the ANOVA test.

\section{RESULTS}

\section{Lesion site}

In all animals the lesion sites were located just ventral to the inferior olive at the caudal end of the medulla oblongata (Fig. $2 A, B)$. The cut transected the superficially located pyramidal tract unilaterally without touching the inferior olive and the medial lemniscus. Transitory effects of the lesion on these deeper structures attributable to edema or inflammation cannot be excluded, however. In all animals CST fibers were seen to retract rostrally from the lesion site and to form retraction bulbs. In control animals almost no local sprouting was seen (Fig. 2C), whereas in IN-1 antibody-treated lesioned animals, massive local fiber outgrowth toward the deeper brainstem structures could be observed (Fig. 2D). Some fibers could be seen to cross or bypass the scar; these fibers were considered to be regenerated CST fibers (O. Raineteau, W. J. Z'Graggen, M. Thallmair, and M. E. Schwab, unpublished observations).

The lesion site of animals that underwent a second lesion was located $1 \mathrm{~mm}$ rostral to the first and showed similar findings (Fig. 2A).

\section{Antibody treatment}

The antibody-producing hybridoma cells implanted in the hippocampus formed small, local cell aggregates in the tissue and the lateral ventricle. Staining with labeled anti-mouse antibody showed high levels of mouse antibodies in the vicinity of the transplants, strong staining of the brain surface and the ventricles, and a gradient of staining into the CNS tissue including the ruber nuclei and the pons. These results were confirmed by ELISA tests (data not shown).

\section{Behavioral tasks}

\section{Food pellet reaching task}

Quantitative analysis of reaching: time, number of attempts, and success rate. The time to grasp 20 stabilized pellets from the shelf was recorded (Figs. 3, 4A). The preoperative baseline measurement showed no differences between the future surgical groups: the mean time to grasp and eat 20 pellets was 75 sec. Forty-two days after operation, the lesion-only as well as the anti-HRPtreated, lesioned animals showed a large increase in time measurements, to a mean of $150 \mathrm{sec}$ (Fig. $4 A$ ). In contrast, the values of lesioned and mAb IN-1-treated animals $(75 \mathrm{sec})$ were indistinguishable from the preoperative values and sham-operated animals.

The number of attempts, indicated by extension of the impaired forelimb through the opening (Fig. 3) to obtain one pellet in the 20-pellet paradigm, is illustrated in Figure $4 B$. Relative to baseline measurements (1.5 attempts per pellet), the number of attempts increased to five per pellet in lesion-only animals and to 4.5 per pellet in anti-HRP-treated, lesioned animals at $42 \mathrm{~d}$ after surgery. In contrast, the mAb IN-1-treated, lesioned animals grasped a pellet after two attempts, which is not significantly different from preoperative values or sham-operated, antibodytreated animals (Fig. 4B). A time course in weekly intervals (Fig. $4 C$ ) shows a significant difference of lesion-only or anti-HRPtreated, lesioned animals 2 weeks after operation compared with baseline or sham-operated, antibody-treated, or mAb IN-1treated, lesioned animals. This difference persisted throughout the entire testing period.

The number of pellets grasped and eaten (excluding dropped and lost ones) was defined as the success rate in the 20-pellet task. The preoperative success rate was 19 out of 20 pellets for all groups (Fig. 4D). Postoperative success rates $42 \mathrm{~d}$ after operation were decreased significantly to $\sim 16$ pellets in the lesion-only and anti-HRP-treated, lesioned rats. In contrast, mAb IN-1-treated, lesioned animals again showed normal values for the success rate, identical to baseline values and sham-operated, antibody-treated controls.

Qualitative analysis of movement components. Nine specific movement components were assessed from video recordings using the previously described four-point disability score system (Whishaw et al., 1993). The ratings of seven individual acts comprising a reaching movement $42 \mathrm{~d}$ after operation are represented in Figure $5 A$. The components of digit opening and pronation were not affected by the lesion and therefore are not shown. The lesion-only and anti-HRP-treated, lesioned animals showed persistent impairment of initiation of the movement, 

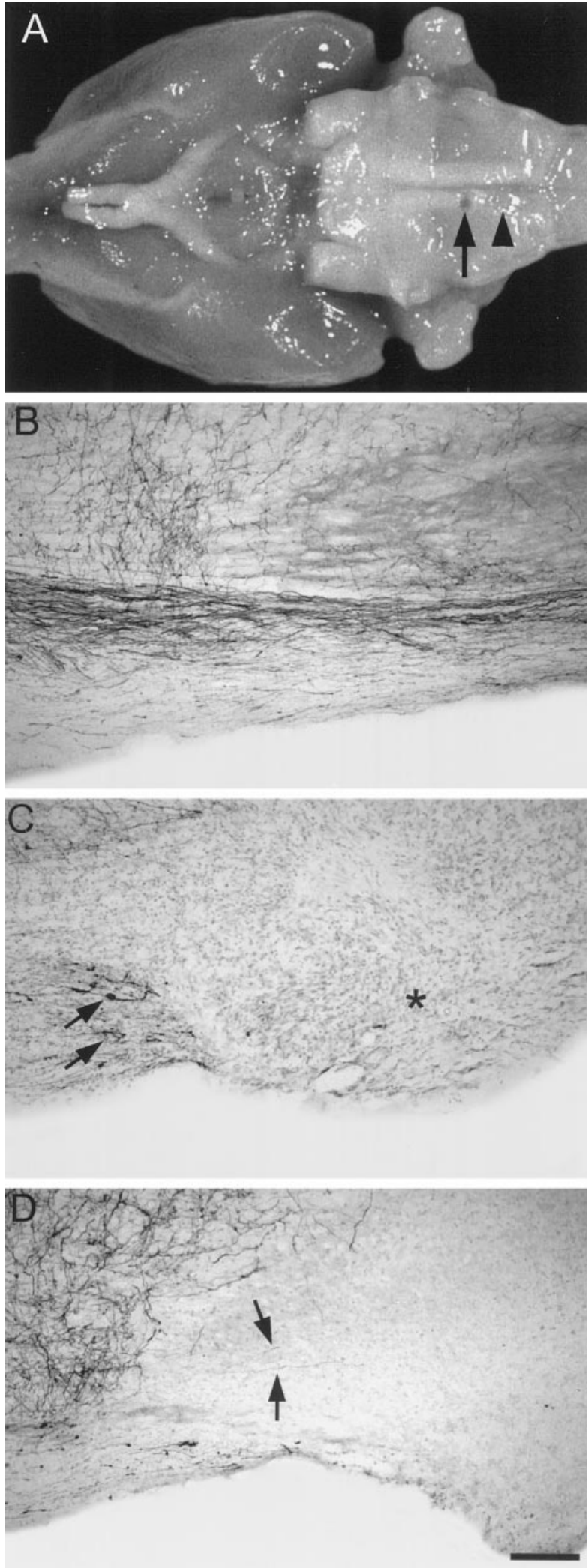

Figure 2. A, Photograph of a brain from ventral illustrating the lesion sites of the first (arrowhead) and second (arrow) pyramidal tract section. $B$, Photomicrograph of longitudinal section through the caudal medulla oblongata and the lesion site of an unlesioned, control antibody-treated animal after a survival time of 16 weeks showing the intact CST. $C$, Lesion site of an animal without antibody treatment after a survival time of 16 weeks. Scar tissue has filled in the original lesion site (star). The

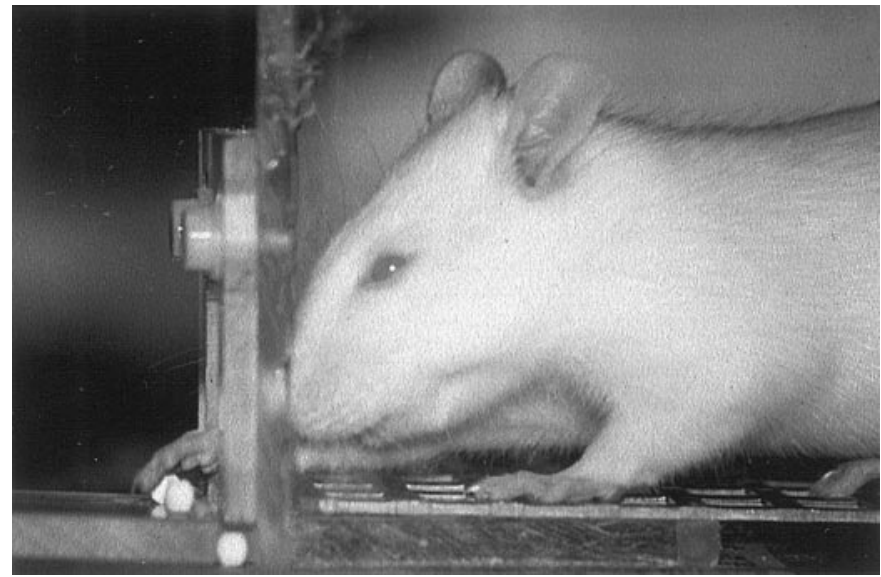

Figure 3. Food pellet reaching task. Animals were biased to grasp small food pellets with the forelimb contralateral to the lesion, the impaired side, through an opening in the wall of a transparent Plexiglas box.

aiming, advance, grasp, supination, food pellet release, and stop of the movement. Lesion-only and anti-HRP-treated, lesioned animals manifested a hesitation or even complete inability of movement initiation. In the aiming and advance components, these animals revealed ataxia and showed a reduced extension of the impaired forelimb. In supination, food pellet release, and movement stop, both groups again showed high impairment scores. The inability to stop movements appropriately was evidenced by difficulties in bringing the paw to the mouth. This deficit was compensated by different strategies, mainly head movements following the paw and turning to the impaired side to extract the pellet.

In contrast, mAb IN-1-treated, lesioned animals showed no deficits in movement initiation, aiming, advance, and grasp (Fig. $5 A$ ). However, in supination, food pellet release, and movement stop, a permanent significant impairment occurred as compared with preoperative values and with sham-operated, antibodytreated animals. This impairment was significantly smaller than that of the other operated groups (Fig. $5 A$ ).

The postoperative time course of the sum of all movement component scores is illustrated in Figure $5 B$. During the entire testing period the disability scores of lesion-only and anti-HRPtreated, lesioned animals were significantly higher than the values of sham-operated, antibody-treated animals. In contrast, mAb IN-1-treated, lesioned animals showed minor impairments compared with baseline and sham-operated animals. All groups showed small improvements over time within the testing period.

\section{Rope climbing}

Foot slips of the impaired forelimbs and hindlimbs that occurred during rope climbing were counted from videotapes and expressed as number of foot slips per step. In the baseline measurements of all groups, nearly no foot slips occurred (Fig. 6A). At postoperative day 21 , anti-HRP-treated, lesioned animals made a mean of 4.5 foot slips per 10 steps as compared with 1 error per

lesioned CST fibers have retracted rostrally and have formed retraction bulbs (arrows). D, Lesion site of an animal treated with the mAb IN-1 after a survival time of 16 weeks. Local fiber sprouting toward the deeper brainstem structures has occurred. The arrows indicate regenerated fibers, passing into the scar tissue. Scale bar, $140 \mu \mathrm{m}$. Magnification $70 \times$. Rostral is to the left in $A-D$. 
A

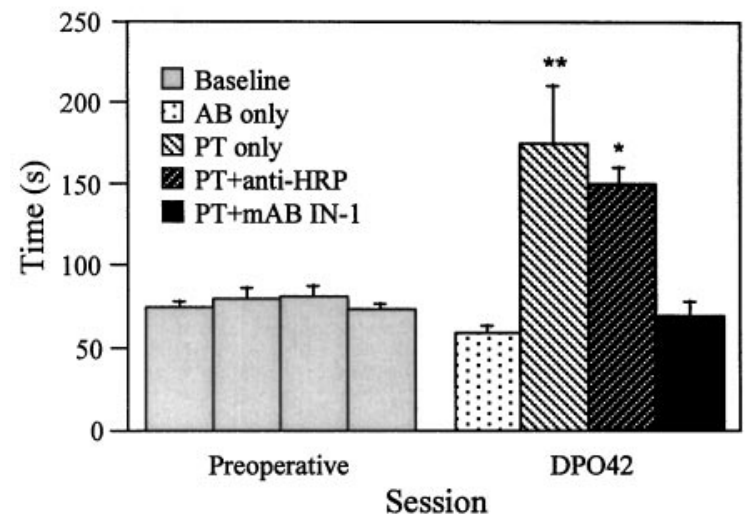

C

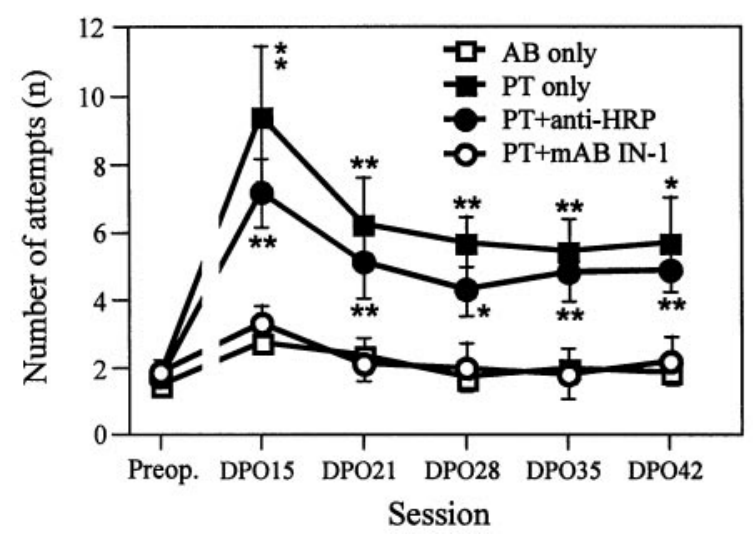

B

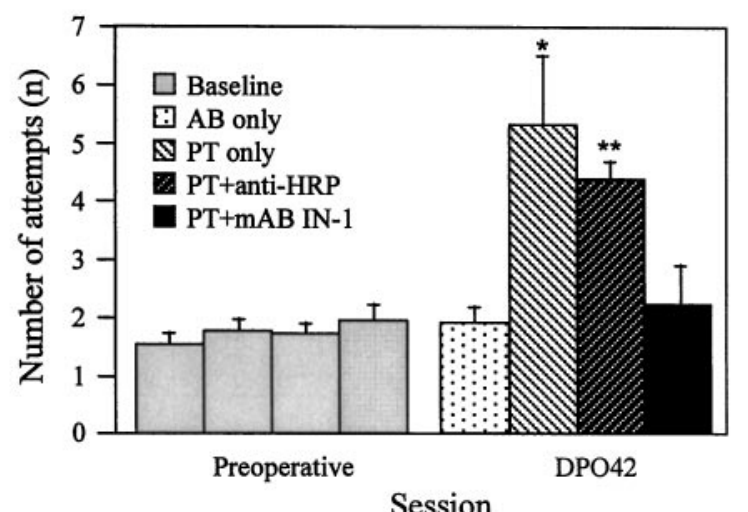

D

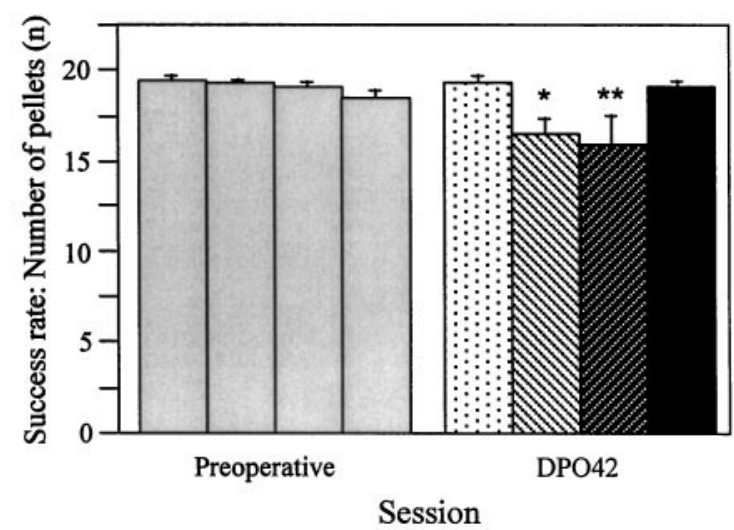

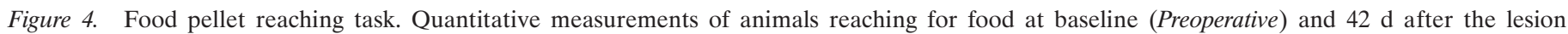

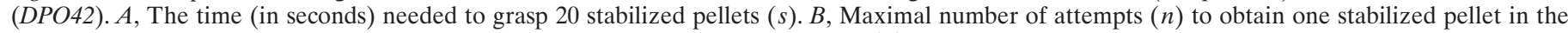

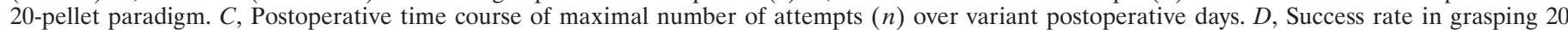

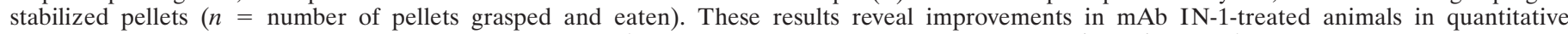

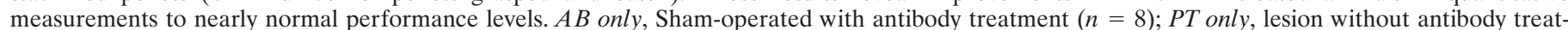

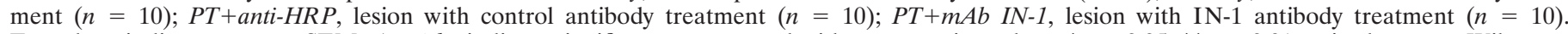

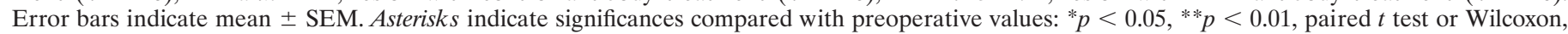
respectively.

10 steps in the sham-operated group (Fig. 6A). Lesioned and mAb IN-1-treated rats showed almost normal performance, statistically indistinguishable from that of sham-operated animals. Compared with baseline, all experimental animals made more foot slips in the postoperative testing sessions, possibly because of increased body weight.

Measurements of the time necessary to climb the rope showed no differences among the experimental groups, thus excluding influences of the climbing speed on the number of foot slips.

\section{Grid walk}

The performance of the animals on a grid runway with irregularly spaced bars, changing from test to test, was analyzed from weekly video recordings by counting the number of foot falls per step of forelimbs and hindlimbs of both sides. The results are shown in Figure $6 B$. Before operation, the rats made $<1$ error per 10 steps. On postoperative day 42, lesioned and lesioned and anti-HRPtreated animals had a significantly increased error rate (approximately two errors/10 steps). Lesioned, mAb IN-1-treated rats, in contrast, showed normal error rates (mean of 0.9 errors/10 steps). The time measurements revealed no differences between the experimental groups, thus excluding influences of the walking speed on the number of foot falls.

\section{Functional maintenance after relesioning}

To analyze the possible contribution to functional recovery of fibers regenerated from the cut axons into the spinal cord, the already lesioned CST was transected a second time, $1 \mathrm{~mm}$ rostral to the first lesion. Two weeks later, animals were retested for food pellet reaching, rope climbing, and grid walk for another 2 weeks. The analysis of the final time measurements in the food pellet reaching task, 4 weeks after the second lesion, is illustrated in Figure $6 C$. In most measurements, lesion-only and anti-HRPtreated, lesioned animals revealed performance levels closely comparable to the performance at day 42 after the first lesion, i.e., before relesioning. The same occurred in mAb IN-1-treated, lesioned animals: no statistical difference in their performance before relesioning was observed. The measurements in the rope climbing and the grid walk test also reflected no loss of the functional recovery in the $\mathrm{mAb}$ IN-1-treated, lesioned group after the second lesion (data not shown). 


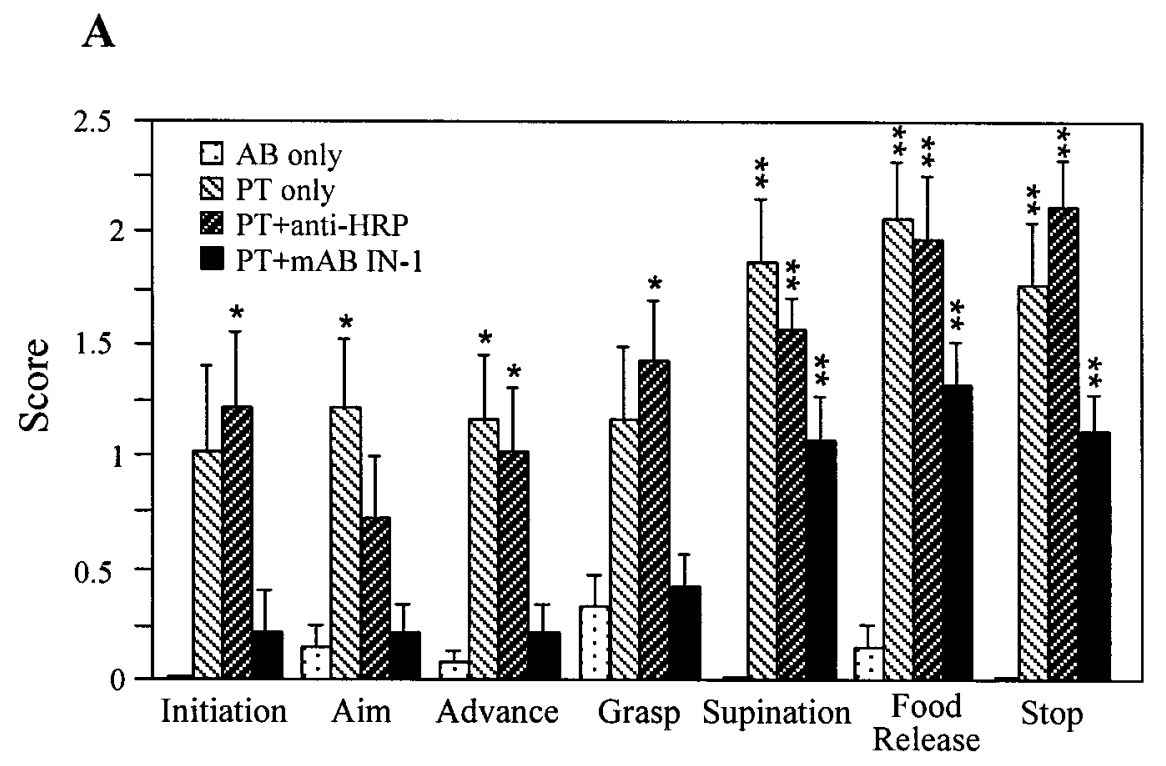

\section{B}

Figure 5. Food pellet reaching task: disability scores. Qualitative analysis of single components comprising a reach for all experimental groups. $A$, Individual movement categories on postoperative day 42. A score of 0 indicates normal forelimb performance; a score of 3 indicates absent movements (for more detail, see Results). B, Time course of combined scores from baseline (Preoperative) through weekly postoperative intervals $(D P O=$ days postoperative $)$. The qualitative analysis of the reaching movement in $\mathrm{mAb}$ IN-1-treated animals shows marked improvement in most movement components. $A B$ only, Sham-operated with antibody treatment $(n=8)$; $P T$ only, lesion without antibody treatment $(n=$ 10); PT+anti-HRP, lesion with control antibody treatment $(n=10) ; P T+m A b I N-1$, lesion with IN-1 antibody treatment $(n=10)$. Error bars indicate mean \pm SEM. Asterisks indicate significances compared with preoperative values: ${ }^{*} p<$ $0.05,{ }^{* *} p<0.01$, Wilcoxon.

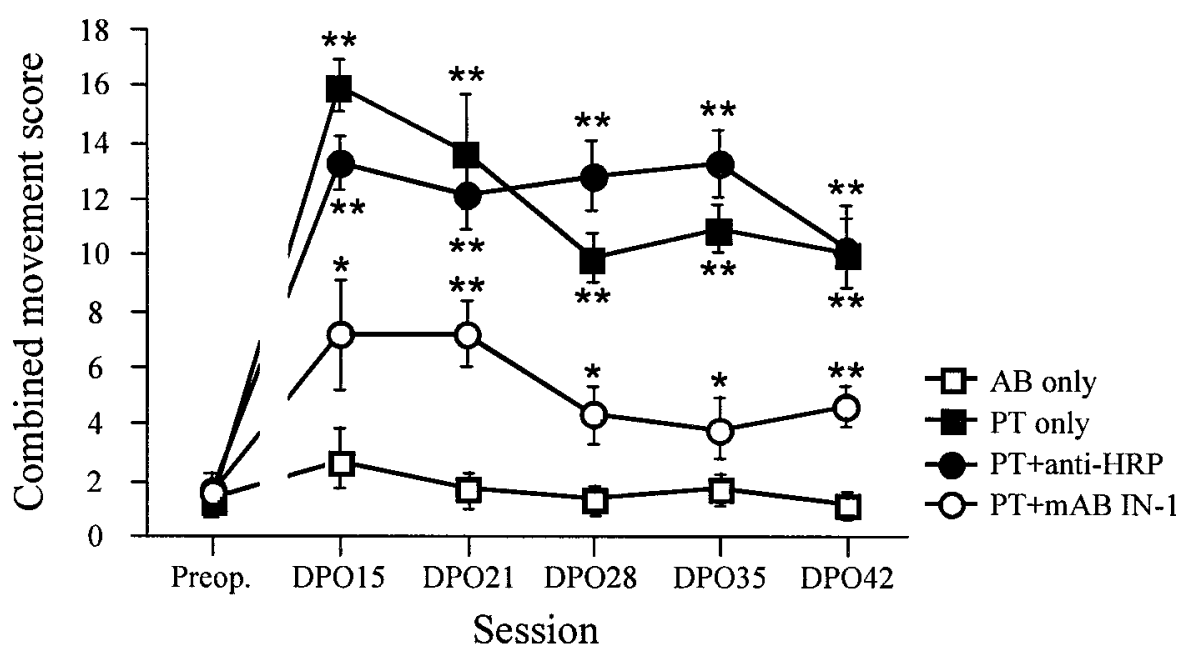

\section{Neuroanatomical analysis}

\section{Cortical injection sites}

The examination of the injection sites showed the characteristic features of a BDA-injection site as described previously (Brandt and Apkarian, 1992; Jiang et al., 1993), with some differences between iontophoretic injections and pressure injections. The five iontophoretic injections into the primary motor cortex resulted in a well localized, small deposition of the tracer with minor spread, centered in layer $\mathrm{V}$ of the primary motor cortex. More extensive spread into surrounding layers was seen after pressure injections, but not into the deep white matter or subcortical structures. The overall size of the labeled cortical areas was quite similar within the two methods. The iontophoretic injections after intracortical microstimulation were restricted to the caudal forelimb area of the primary motor cortex, with minor co-labeling of other areas, whereas BDA pressure injections may have resulted in some labeling of the neighboring face and hindlimb areas.

\section{Tracing}

The analysis of the cerebral peduncle at the pontine level showed a typical and consistent distribution of BDA-positive fibers within the peduncle on cross sections. Independent of the antibody treatment and the lesion, most of the labeled fibers were situated in the medial half of the cerebral peduncle, whereas only a minor part was seen in the lateral half, as described earlier (Mihailoff et al., 1978; Kosinski et al., 1986). The average number of BDApositive fibers at midpontine level was $5280( \pm 336 \mathrm{SEM} ; n=24)$ for the animals traced by iontophoretic injections and was almost identical to the number in the pressure-injected group (5210 \pm 355 SEM; $n=26$ ).

\section{Corticorubral projection}

The anatomy of the ipsilateral corticorubral projection in all experimental groups showed a similar pattern. At midbrain levels, the labeled fibers left the cerebral peduncle on its dorsal aspect, passed the substantia nigra, where some fibers were observed to 


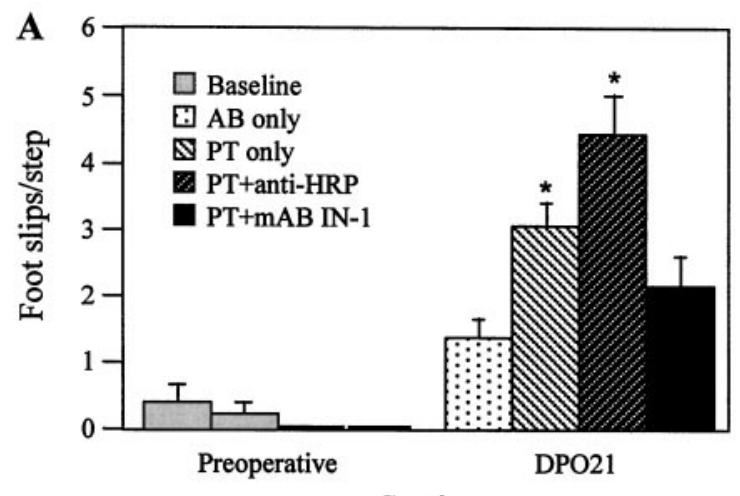

Session
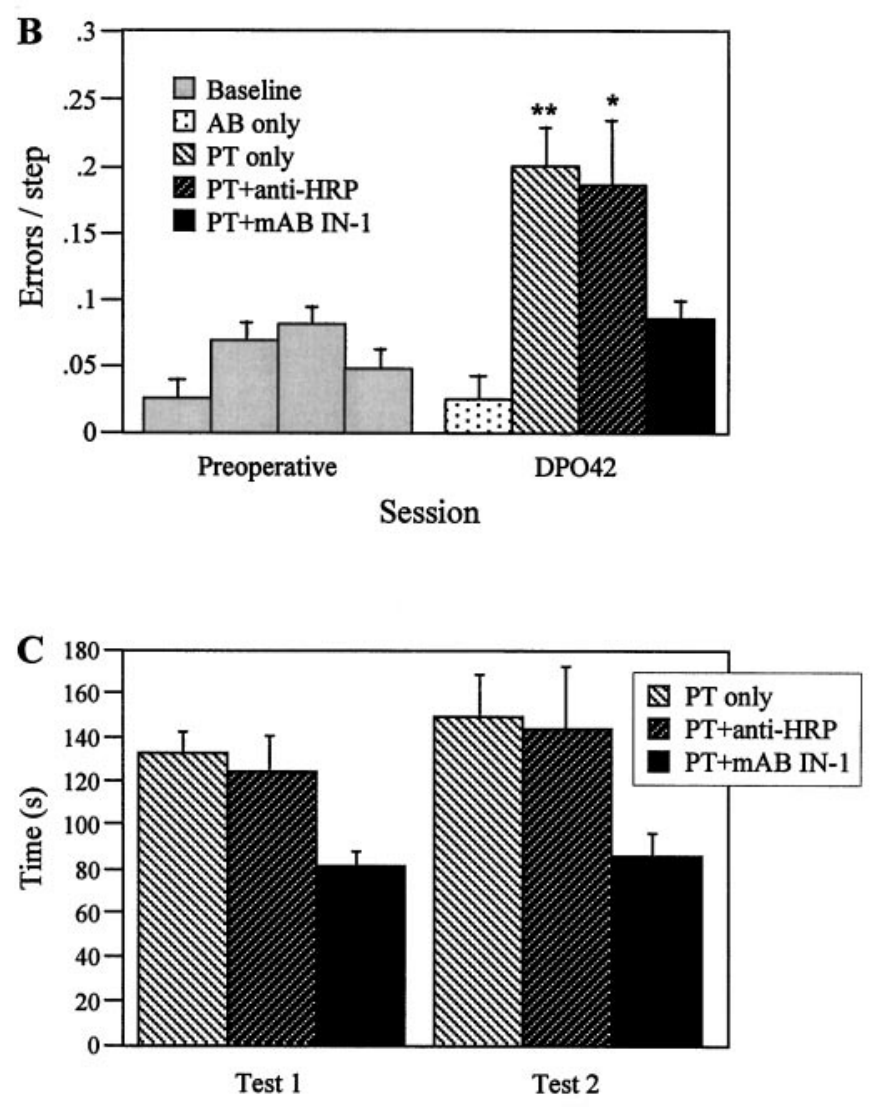

Figure 6. A, Rope climbing. The animals had to climb a vertical rope. The number of foot slips per step was measured at baseline (Preoperative) and $21 \mathrm{~d}$ after the lesion (DPO21). This test revealed improved grip strength in mAb IN-1-treated animals as compared with lesioned control groups. $B$, Grid walk. The ability of the animals to cross a grid with randomly assigned gaps was recorded. The performance was analyzed in the ratio of the number of errors per step at baseline (Preoperative) and $42 \mathrm{~d}$ postoperatively (DPO42). Lesioned animals showed improved limb coordination and accurate limb placement after mAb IN-1 treatment. $C$, Food pellet reaching task. The time to grasp 20 stabilized pellets at day 42 after the first lesion (Test 1) and 4 weeks after relesioning the pyramidal tract slightly rostral to the first lesion site (Test 2). mAb IN-1-treated animals showed no impaired performance after the second lesion. $A B$ only, Sham-operated with antibody treatment $(n=8)$; PT only, lesion without antibody treatment $(n=10$; Fig. $6 C, n=4) ; P T+$ anti-HRP, lesion with control antibody treatment $(n=10$; Fig. $6 C, n=4)$; $P T+m A b I N-1$, lesion with IN-1 antibody treatment $(n=10$; Fig. $6 C: n=4)$. Error bars indicate mean \pm SEM. Asterisks indicate significances compared with preoperative values: ${ }^{*} p<0.05,{ }^{*} p<0.01$, Wilcoxon. terminate and form bouton-like endings, and then turned sharply medial, toward the nucleus ruber. There, the fibers emanating from the cerebral peduncle were joined by fibers that had descended through the thalamus. As described earlier by others for the rat (Brown, 1974; Gwyn and Flumerfelt, 1974; Flumerfelt, 1980; Naus et al., 1985a,b), the axons terminated primarily in the parvocellular part of the ipsilateral red nucleus, in the pararubral area, in the prerubral field, and around the fasciculus retroflexus. A few BDA-labeled fibers were also seen to terminate in the magnocellular portion of the ipsilateral nucleus ruber with bouton-like endings. In normal control animals, a few fibers crossed the midline and terminated in the contralateral nucleus ruber, primarily in the parvocellular region. Rats with pyramidal tract lesion and no treatment or control antibody treatment were indistinguishable from these normal animals (Fig. 7A,C).

In the animals that underwent pyramidotomy and treatment with $\mathrm{mAb}$ IN-1, the same ipsilateral corticorubral projection pattern could be observed as in the control groups. In contrast to controls, however, significantly more BDA-positive fibers were seen to cross the midline and to innervate the contralateral nucleus ruber (Fig. $7 B, D$ ). The counting of the midline crossing fibers showed 45-140 fibers in the control groups and 180-230 fibers in the lesioned IN-1 antibody-treated group of animals. The number of each animal was divided by the total number of labeled CST fibers to correct for the differences in the tracing. The results are shown in Figure 8. After a survival time of 2 weeks as well as after 16 weeks, the relative number of midline crossing fibers was much higher in the IN-1 antibody-treated groups than in normal animals, in sham-operated animals, or in lesioned and control antibody-treated rats. These crossing axons originated from the ipsilateral red nucleus or seemed to bypass it dorsally. Some of the fibers crossed the midline directly toward the contralateral nucleus ruber, whereas others crossed more dorsally in the central gray (Fig. $7 B, D$ ). These axons ended in the area of the contralateral nucleus ruber, mainly in the parvocellular part; only a few axons seemed to end in the magnocellular part.

\section{Corticopontine projection}

The cortical projection to the ipsilateral pons from the forelimb area in all groups examined showed the typical topographical innervation pattern as described earlier (Mihailoff et al., 1978; Wiesendanger and Wiesendanger, 1982; Rouiller et al., 1993; Panto et al., 1995). Labeled fibers running in the cerebral peduncle over the basilar pons left the peduncle ventrally and formed typical termination fields. At rostral levels, a dense termination field was observed centrally. At midpontine levels, this termination field was split into a small medial and lateral area. In addition, a new termination zone dorsal to the cerebral peduncle appeared. At caudal levels the termination zone enlarged again, and extensive labeling was observed in the medial, ventral, and lateral pons and around the ventral and dorsal aspect of the cerebral peduncle. In normal rats and in all the control groups a very minor contralateral component, mainly at midpontine to caudal pontine levels, was present.

Although the ipsilateral corticopontine projection was not distinguishable in all the treatment groups from that of normal, untreated animals (see Fig. 10C,D), lesion and mAb IN-1 treatment resulted in a very marked increase in the density of innervation to the contralateral basilar pontine nuclei (Figs. 9B, $D$, $10 A, B)$. Densitometry of the terminal fields showed a significant difference of the contralateral in relation to the ipsilateral fiber density: $26.3 \%( \pm 2.6 \mathrm{SEM} ; n=5)$ in the lesioned IN-1 antibody- 

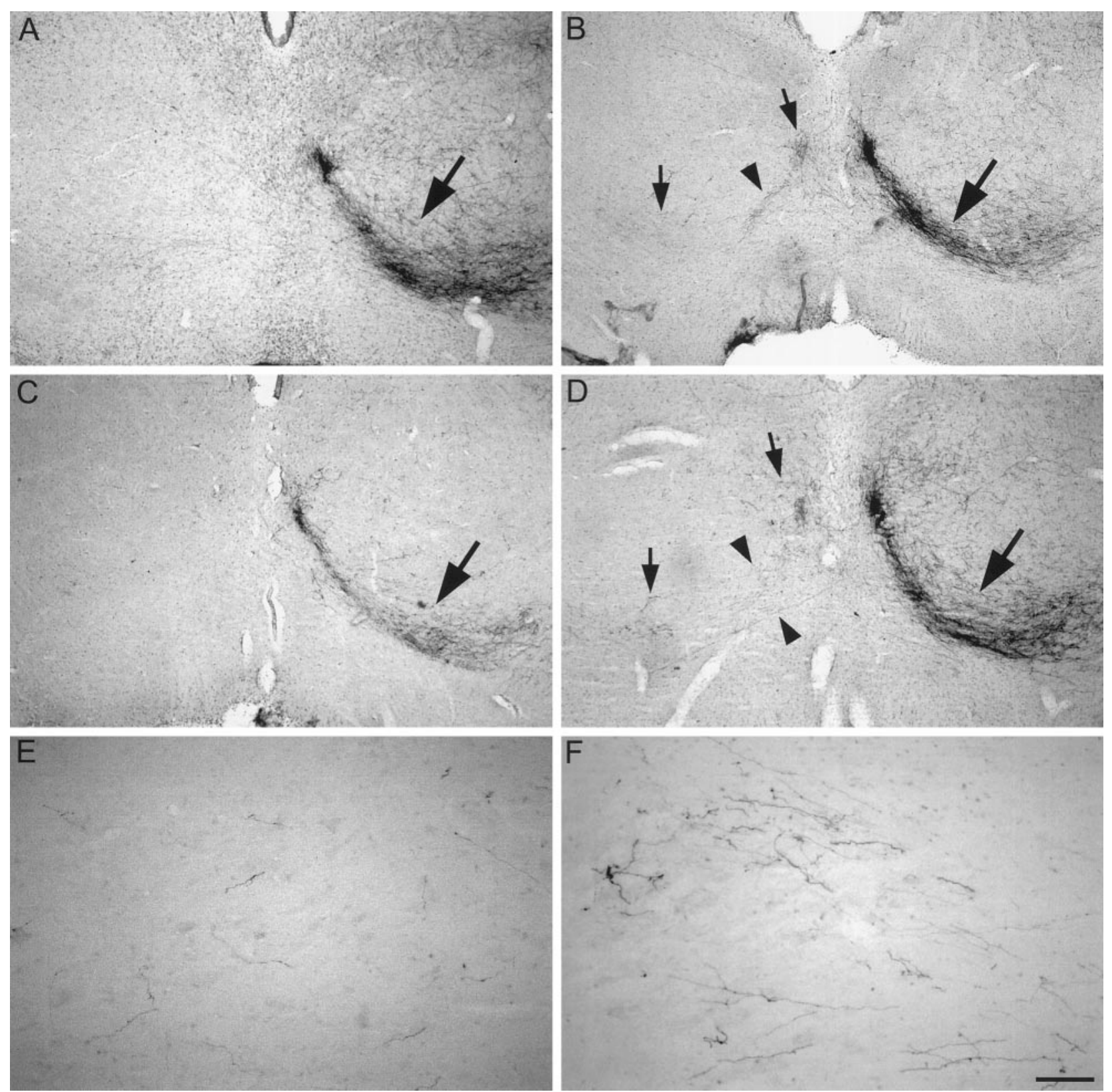

Figure 7. Cross sections of the nucleus ruber. $A$, Corticofugal projection to the nucleus ruber of a lesioned animal treated with control antibody for 2 weeks. The axons terminate primarily ipsilaterally (large arrow); only a sparse, barely visible contralateral termination exists. $B$, mAb IN-1-treated animal, 2 weeks after lesion. Significantly more fibers cross the midline and terminate in the contralateral nucleus ruber (small arrows). $C$, Control antibody-treated animal 16 weeks after pyramidotomy. Only a few fibers cross the midline and innervate the contralateral parvocellular nucleus ruber. $D$, IN-1 antibody-treated animal after a survival time of 16 weeks. Many axons (arrowhead) crossing the midline and ending in the contralateral red nucleus are seen (small arrows). The contralateral termination fields mirror the ipsilateral side. E, Higher-power photomicrograph of the contralateral parvocellular red nucleus of a control antibody-treated animal (16 weeks). Only a few crossed fibers are present. $F$, Contralateral nucleus ruber of an IN-1 antibody-treated animal (16 weeks). Many fibers branch and terminate with bouton-like endings in the parvocellular nucleus ruber. Scale bars: $A-D, 280$ $\mu \mathrm{m} ; E, F, 70 \mu \mathrm{m}$. Magnification: $A-D, 35 \times ; E, F, 140 \times$.

treated animals compared with $14.6 \%( \pm 2.6 ; n=5 ; p<0.05$ in ANOVA) in the control antibody-treated group after 2 week survival. After a 16 week survival time, similar results were obtained: $15.9 \%( \pm 3.0 \mathrm{SEM} ; n=5)$ contralateral labeling in the IN-1 antibody-treated group compared with $3-6 \%$ in the different control groups (Fig. 10A,B). Interestingly, this increased bilateral projection remained precisely in the confines of the forelimb termination zones, thus reflecting the normal ipsilateral projection (Fig. 9E). The differences of the percent values be- tween the 2 week and 16 week groups are probably attributable to the slightly different labeling procedures used, as described above. Small differences in the topographical innervation pattern were also observed, depending on the tracing method.

Counting of the fibers crossing the midline divided by the total number of labeled CST fibers to correct for the interanimal differences in the tracing showed no differences at the rostral and midpontine levels but a significant increase in the caudal quarter of the pons: $327.1 \times 10^{-4}\left( \pm 33.2 \times 10^{-4} \mathrm{SEM} ; n=6\right)$ in the 


\section{Nucleus Ruber: crossing fibers}
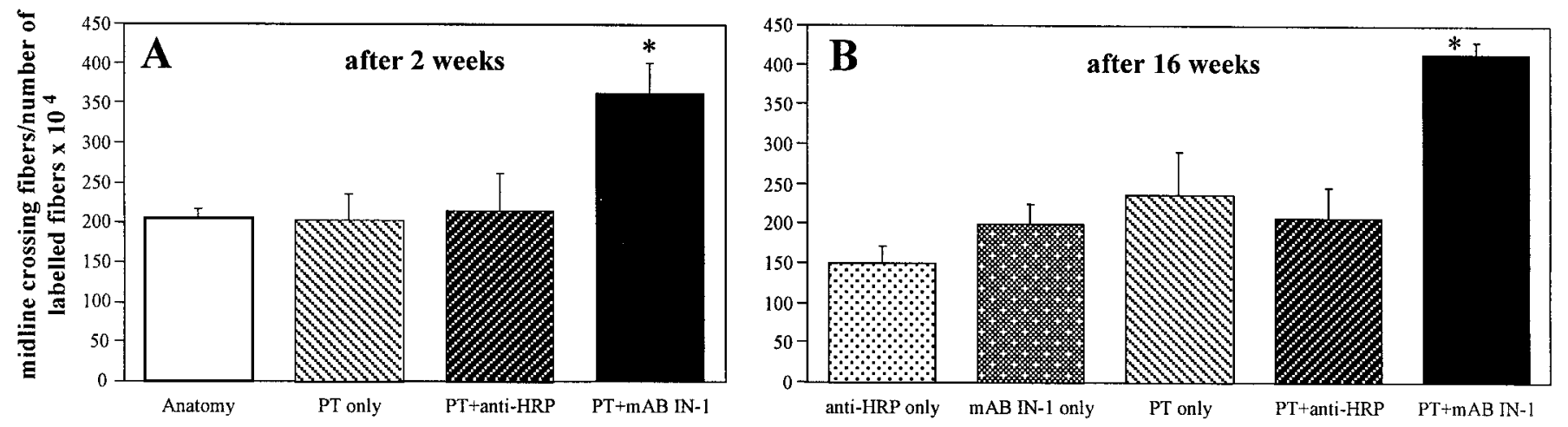

Figure 8. Number of midline crossing fibers in the area of the parvocellular nucleus ruber divided by the total number of labeled CST fibers, to correct for the differences in the tracing. The asterisk indicates significance (ANOVA; $p<0.05$ ). $A$, Survival time of 2 weeks. $B$, Survival time of 16 weeks.

mAb IN-1-treated group compared with $147 \times 10^{-4}( \pm 41 \times$ $10^{-4} \mathrm{SEM} ; n=5 ; p<0.05$ in ANOVA) in the control antibodytreated group after 2 weeks; $506.5 \times 10^{-4}\left( \pm 78.2 \times 10^{-4}, n=5\right)$ in the IN-1 antibody-treated group compared with $283.3 \times 10^{-4}$ $\left( \pm 31.2 \times 10^{-4} \mathrm{SEM} ; n=6 ; p<0.05\right.$ in ANOVA) in the group treated with control antibody after 16 weeks. The fibers were seen to cross the midline either in the ventral part of the pons (white matter) or directly between the basilar pontine nuclei (gray matter). These data show an increase of corticopontine fibers crossing the midline and an increase in the density of terminal fibers in response to pyramidotomy and $\mathrm{mAb} \mathrm{IN}-1$ treatment.

\section{DISCUSSION}

The major findings of this study may be summarized as follows. The monoclonal antibody IN-1, which is known to neutralize a major component of the inhibitory activity of CNS myelin, resulted in a high degree of functional recovery in skilled forelimb movements and grip strength after unilateral pyramidotomy in adult rats. mAb IN-1 treatment also enhanced specific corticorubral and corticopontine plasticity in these rats. Thus, the hemicortex that had lost its access to the spinal cord by the lesion established a bilateral innervation of specific, anatomically correct parts of the red nucleus and the basilar pons.

\section{mAb IN-1 treatment leads to almost complete functional recovery after pyramidotomy}

Our results demonstrate almost full recovery in skilled forelimb movements after pyramidotomy and mAb IN-1 treatment. The overall time to grasp and eat 20 food pellets, the number of attempts to obtain one pellet, and the success rate in reaching normalized completely to preoperative values in these animals. In contrast, animals that underwent pyramidotomy without antibody treatment or animals with lesion and control antibody treatment were highly and permanently impaired in all these tests.

The qualitative analysis of the movement components during a reaching act showed almost full recovery in movement initiation, aiming, advancing, and grasp of a food pellet after mAb IN-1 treatment after lesion. In contrast, the movement components supination of the paw, digit extension for food release, and movement stop also remained impaired in the mAb IN-1-treated, lesioned animals, but the impairment was significantly smaller compared with lesioned control groups. mAb IN-1 treatment also led to recovery of grip strength during rope climbing in lesioned animals $21 \mathrm{~d}$ postoperatively (later time points gave aberrant results, especially in male rats, because of increased body weight).

All of the results from our control lesioned rats are well in line with the literature. Chronic impairments in complex digital functions and movement accuracy, changes in limb preference, and deficits in the ability to make rotary limb movements after unilateral pyramidal tract lesion were described in rats and hamsters (Castro, 1972; Reh and Kalil, 1982; Whishaw and Kolb, 1988; Whishaw et al., 1993). In monkeys, deceleration of movements after a CST lesion, which induced prolonged time requirements in reaching tasks, was shown (Hepp-Reymond and Wiesendanger, 1972).

Deficits seen in the grid walk paradigm in lesioned control animals may be attributable to problems with movement precision as well as disturbed coordination between both forelimbs and hindlimbs after CST lesion. This observation is supported by previous findings of persistent hypermetria in hindlimb movements after pyramidotomy (Metz et al., 1998). mAb IN-1 treatment of lesioned animals resulted in complete recovery in this test, also indicating recovery in hindlimb movements and interlimb coordination under these conditions.

\section{The monoclonal antibody IN-1 enhances corticorubral and corticopontine plasticity}

Our results demonstrate an increase in the number of corticorubral axons originating in the area of the forelimb motor cortex crossing the midline between the ipsilateral and contralateral nucleus ruber after treatment with the mAb IN-1 and unilateral pyramidotomy. These fibers terminate in the contralateral parvocellular nucleus ruber and form bouton-like structures that suggest synaptic terminals. Some crossing fibers could be identified as collaterals of corticorubral axons with extensive ipsilateral terminations. It can be assumed, therefore, that our findings are at least in part the result of sprouting and outgrowth of collaterals to the contralateral parvocellular red nucleus from ipsilateral corticorubral axons.

Earlier studies of the corticorubral projections in the rat (Brown, 1974; Gwyn and Flumerfelt, 1974; Flumerfelt, 1980; Naus et al., 1985a,b) describe a dense, exclusively ipsilateral innervation from the primary motor cortex, restricted to the parvocellular region of the red nucleus. Our results confirm this earlier work, but we also found a very minor contralateral innervation, restricted to the parvocellular part. Such a crossed corti- 

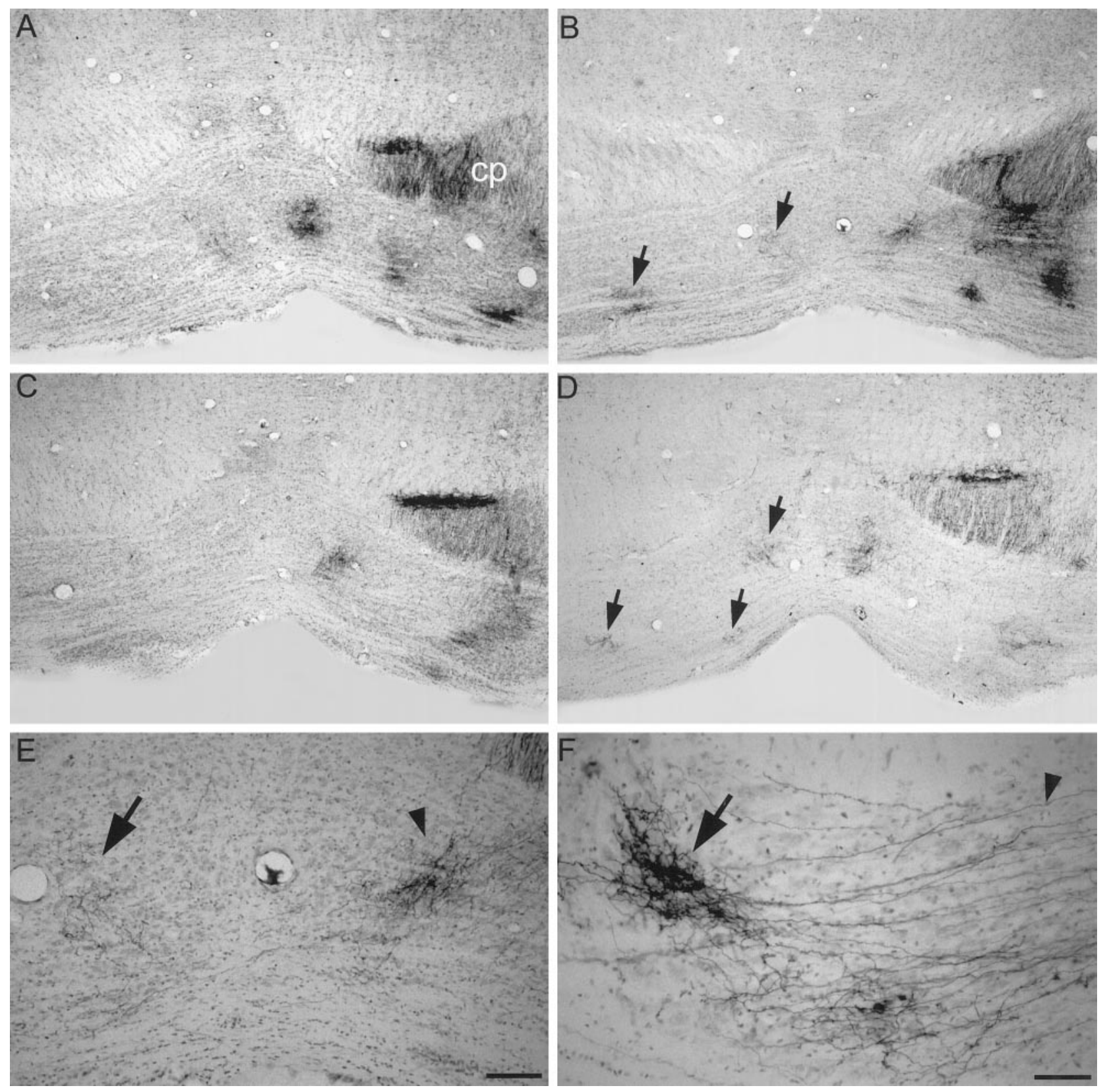

Figure 9. Cross sections at midpontine level. $A$, Control antibody-treated animals 2 weeks after pyramidotomy. BDA-positive fibers leave the cerebral peduncle $(c p)$ ventrally and form typical termination zones, almost completely restricted to the ipsilateral basilar pontine nuclei. Only few fibers end on the contralateral side close to the midline. $B$, Animal treated with the mAb IN-1 2 weeks after lesion. An increase in the innervation of the contralateral basilar pontine nuclei can be noted (arrows), whereas the ipsilateral side seems unchanged. $C$, Animal treated with control antibody (16 weeks). $D$, IN-1 antibody-treated animal (16 weeks). Similar to the findings after 2 weeks, enlarged contralateral termination fields can be observed (arrows). The ipsilateral projection is unchanged. E, Higher-power photomicrograph of Figure $2 B$ showing the midline and the ipsilateral (arrowhead) and contralateral (arrow) innervation. $F$, Crossing fibers (arrowhead) and arborization (arrow) in the contralateral pons of an IN-1 antibody-treated animal after 2 week survival time. Scale bar: $A-D, 280 \mu \mathrm{m} ; E, 140 \mu \mathrm{m} ; F, 70 \mu \mathrm{m}$. Magnification: $A-D, 35 \times ; E, 70 \times ; F, 140 \times$.

corubral projection has been described for the cat (Murakami and Higashi, 1988). A possible explanation for these differences might be the higher sensitivity and anatomical resolution of BDA tracing as compared with the Fink-Heimer's and other tracing methods used in the earlier studies.

The treatment with the mAb IN-1 also resulted in an increase in the number of fibers crossing the midline in the pons and of the density of the contralateral innervation of the basilar pontine nuclei after pyramidotomy. These new contralateral terminations were specific for the forelimb areas, thus mirroring the ipsilateral side, which receives a somatotopic projection from the motor cortex ending in a typical four columnar innervation pattern (Mihailoff et al., 1978; Wiesendanger and Wiesendanger, 1982; Rouiller et al., 1993; Panto et al., 1995). In agreement with earlier studies, a very minor contralateral projection was also found in the normal animals. Similar to the ipsilateral corticorubral projection, the density of the ipsilateral corticopontine termination was unchanged after lesion and treatment with the IN-1 antibody.

The described corticorubral and corticopontine changes in the IN-1 antibody-treated group were found after a survival time of 


\section{Pons: Densitometry, contralateral projection}
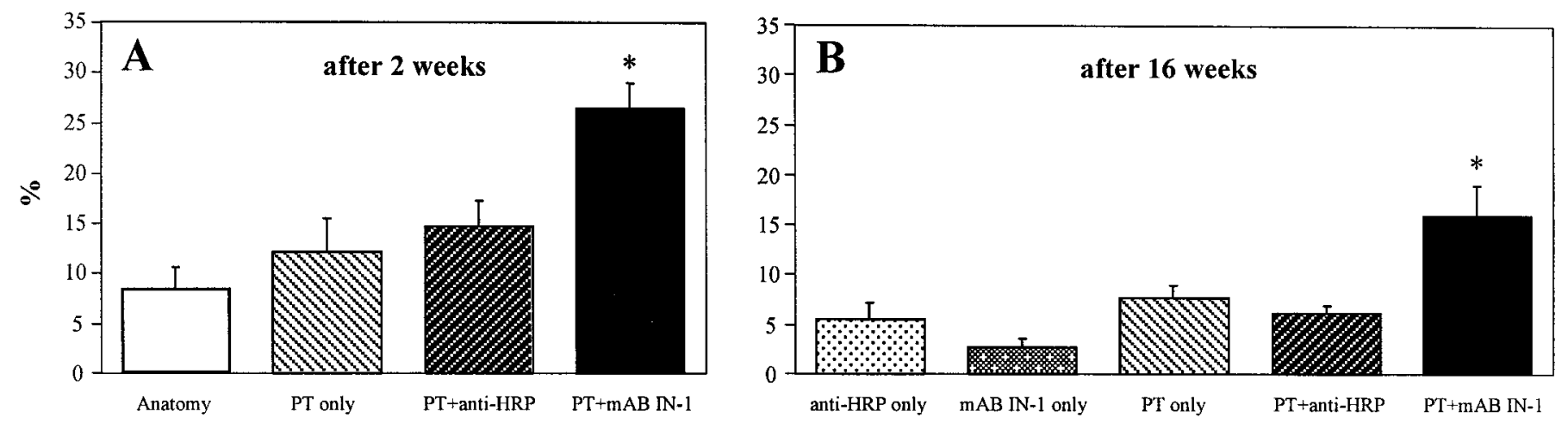

Pons: ipsilateral projection
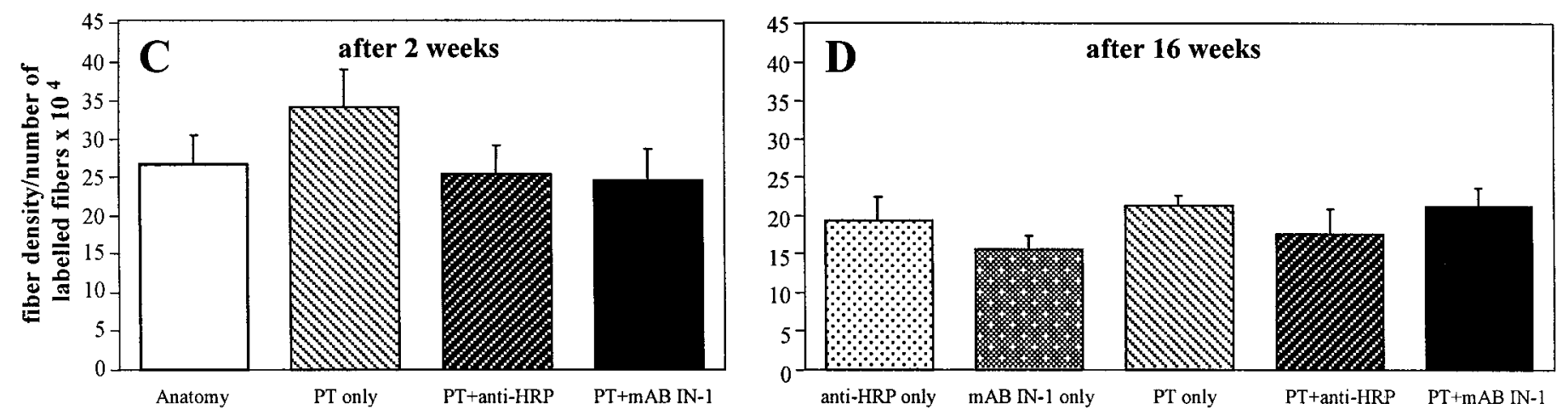

Figure 10. A, B, Densitometry of the terminal fields of corticopontine projections to the contralateral pons, as percent of the ipsilateral side; survival time of 2 and 16 weeks, respectively. The asterisk indicates significance (ANOVA; $p<0.05$ ). $C, D$, Densitometry of the ipsilateral corticopontine innervation, divided by the number of fibers after a survival time of 2 or 16 weeks.

2 weeks as well as after 16 weeks. Although 2 weeks after lesion the antibody-secreting hybridoma xenograph was still macroscopically observable, it could not be detected after 16 weeks (cyclosporin A withdrawal after 14 or $8 \mathrm{~d}$, respectively). Qualitatively and quantitatively, the same changes could be observed after 2 and 16 weeks. The densitometric comparison of the contralateral to the ipsilateral innervation in the pons showed a twofold increase of the contralateral fiber density at 2 weeks and at 16 weeks in the IN-1 as compared with the control groups. We conclude from these findings that sprouting and plastic rearrangement of the corticofugal connections in the ruber and basilar pontine nuclei occur fast, within 2 weeks of treatment with the IN-1 antibody, and remain stable over time also in the absence of the delivery of the antibody.

The sprouting of corticorubral and corticopontine fibers described above did not occur after the application of the mAb IN-1 to sham-lesioned animals, and no changes in any of the behavioral tasks could be observed in these animals compared with baseline values. This indicates that the presence of the IN-1 antibody alone or the local lesion created by the hybridoma transplant in the hippocampus is not sufficient, but that an additional stimulus is needed, probably in the form of the lesion of the CST. The pyramidotomy on its own, however, was also insufficient to trigger corticorubral and corticopontine plasticity, although a tendency (not significant) was observed when lesion-only animals were compared with normal rats. The IN-1 antibody thus creates conditions that allow additional factors to induce sprouting and specific plasticity.

\section{Underlying mechanisms}

After unilateral pyramidotomy in neonatal rats a similar specific corticorubral and corticopontine plasticity was seen without any further treatment (our unpublished results). When one sensorimotor cortex was removed in neonatal rats, the remaining cortex was shown to send bilateral corticofugal projections to the nucleus ruber (Leong and Lund, 1973; Nah and Leong, 1976a,b; Naus et al., 1985a,b) and the basilar pontine nuclei (Leong and Lund, 1973; Castro and Mihailoff, 1983). Although the mechanisms underlying these findings might be different because of the unilateral deafferentation of the subcortical target regions after cortical lesions, which does not occur after pyramidotomy, the neuroanatomical results seem to be similar. Nah et al. (1980) demonstrated that these bilateral projections are newly formed after neonatal lesion and are not the result of persistent neonatal connections. All of these findings suggest the presence of signals that induce sprouting and the establishment of a bilateral, somatotopic innervation pattern.

In the CNS the capacity for plasticity and regeneration decreases during postnatal development (Kuang and Kalil, 1990; Firkins et al., 1993), a process that coincides in time with the formation of myelin (Kapfhammer and Schwab, 1994). The prevention of myelin formation in the spinal cord resulted in the persistence of the sprouting capacity in adult rats (Schwegler et al., 1995; Vanek et al., 1998). The inhibitory effect of CNS myelin on fiber outgrowth, sprouting, and regeneration is caused by the presence of growth inhibitory molecules (Caroni and Schwab, 
1988a; for review, see Schwab and Bartholdi, 1996). The CNS myelin proteins NI-35 and NI-250 play an important role in the inhibitory properties of adult CNS tissue. Neutralization of these molecules with the mAb IN-1 in vitro resulted in successful fiber growth on CNS myelin and in vivo in long distance regeneration of CST axons after bilateral tract transection in adult rats (Schnell and Schwab, 1990, 1993; Bregman et al., 1995). The present data show that growth permissive conditions can be created by the mAb IN-1 in the adult rat brainstem, which contains high levels of myelin in both white and gray matter.

The new sprouting observed in the present experimental paradigm is probably regulated by several factors. Some of the corticorubral and corticopontine projections are collaterals of axons projecting to the spinal cord (Ugolini and Kuypers, 1986; Akintunde and Buxton, 1992). The transection of these axons could lead to compensatory collateral sprouting ("pruning effect") (Schneider, 1973; Devor and Schneider, 1975; Sabel and Schneider, 1988). On the other hand, the pyramidal transection, leading to loss of cortical input to half of the spinal cord, may have induced a functional imbalance of the whole motor system. Activity-regulated local synthesis of chemotropic or neurotrophic factors or of other promotors of sprouting and fiber growth (for review, see Thoenen, 1995; Fagan, 1997) may have induced collateral formation from intact axons, attracted fibers across the midline, or induced terminal axon growth in target fields.

\section{Neuroanatomical plasticity parallels functional recovery}

The mAb IN-1-induced neuroanatomical plasticity occurred fast, within the first 2 weeks after lesion. These processes paralleled the functional recovery seen in $\mathrm{mAb}$ IN-1-treated, lesioned animals, which showed significant functional improvements after 2 weeks. The early time course of recovery during the first 2 weeks postoperatively could not be studied because of the daily cyclosporin A injections, the possible local effect of the hybridoma cell transplant, and its subsequent resorption and the necessary retraining phase. The newly formed neuroanatomical connections in the mAb IN-1-treated, lesioned animals were stable over time, and also the behavioral results of the mAb IN-1 treatment persisted for up to 10 weeks, the latest time point tested. In the control antibody-treated, lesioned rats, small improvements in the behavioral tasks used were seen over 4 weeks of postoperative testing; they were probably caused by a training effect and the development of compensatory mechanisms (Whishaw et al., 1993). In the controls, however, a high degree of functional impairments persisted.

The mAb IN-1 is known to induce long-distance regeneration of lesioned CST axons (Schnell and Schwab, 1990, 1993; Schnell et al., 1994). To answer the question about whether corticofugal plasticity or regeneration of lesioned CST fibers causes the functional improvements seen in the mAb IN-1-treated animals after pyramidotomy, a second lesion was performed rostral to the first, thus transecting possible regenerating fibers. Although some regeneration of injured CST fibers occurred in our rats under the influence of mAb IN-1 (Raineteau, Z'Graggen, Thallmair, and Schwab, unpublished observations), relesioning of the CST in these animals did not lead to reduction of the functional recovery. Increased neuronal plasticity after CST lesions and mAb IN-1 treatment was also observed in the spinal cord (Thallmair et al., 1997). There, collaterals grew out from the intact CST, crossed the midline, and arborized into the denervated part of the spinal cord. It is probable that other fiber systems, which have not been analyzed, also responded by changes in terminal arbors and connectivity to the induced functional imbalance caused by the lesion and the facilitation of growth provided by the presence of the mAb IN-1. Such systems could include other descending motor pathways, sensory systems, or cortical motor or sensory representations. All of them, together with the CNS plasticity described here, may be responsible for the functional recovery observed in our behavioral study.

In conclusion, this study shows that blockade of the myelinassociated neurite growth inhibitors with the mAb IN-1 after unilateral lesion of the CST in adult rats leads to new, specific, bilateral corticorubral and corticopontine projections that are stable over time. In the same animals we observed a very high degree of functional recovery in skilled forelimb reaching, rope climbing, and grid walk. These effects point to interesting future strategies in the treatment of CNS injuries.

\section{REFERENCES}

Akintunde A, Buxton DF (1992) Origins and collateralization of corticospinal, corticopontine, corticorubral and corticostriatal tracts: a multiple retrograde fluorescent tracing study. Brain Res 586:208-218.

Armand J, Kably B (1993) Critical timing of sensorimotor cortex lesions for the recovery of motor skills in the developing cat. Exp Brain Res 93:73-88.

Bandtlow C, Zachleder T, Schwab ME (1990) Oligodendrocytes arrest neurite growth by contact inhibition. J Neurosci 10:3837-3848.

Brandt HM, Apkarian AV (1992) Biotin-dextran: a sensitive anterograde tracer for neuroanatomic studies in rat and monkey. J Neurosci Methods 45:35-40.

Bregman BS, Kunkel-Bagden E, Schnell L, Dai HN, Gao D, Schwab ME (1995) Recovery from spinal cord injury mediated by antibodies to neurite growth inhibitors. Nature 378:498-501.

Brown LT (1974) Corticorubral projections in the rat. J Comp Neurol 154:149-167.

Cao Y, Vikingstad EM, Huttenlocher PR, Towle VL, Levin DN (1994) Functional magnetic resonance studies of the reorganization of the human hand sensorimotor area after unilateral brain injury in the perinatal period. Proc Natl Acad Sci USA 91:9612-9616.

Carlini EA, Teresa M, Silva A, Cesare LC, Endo RM (1967) Effects of administration of $\beta$-(3,4-dimethoxyphenyl)-ethylamine and $\beta$ - $(3,4,5$ trimethoxyphenyl)-ethylamine on the climbing rope performance of rats. Med Pharmacol Exp 17:534-542.

Caroni P, Schwab ME (1988a) Two membrane protein fractions from rat central myelin with inhibitory properties for neurite growth and fibroblast spreading. J Cell Biol 106:1281-1288.

Caroni P, Schwab ME (1988b) Antibody against myelin-associated inhibitor of neurite growth neutralizes nonpermissive substrate properties of CNS white matter. Neuron 1:85-96.

Carr LJ, Harrison LM, Evans AL, Stephens JA (1993) Patterns of central motor reorganization in hemiplegic cerebral palsy. Brain 116:1223-1247.

Castro AJ (1972) Motor performance in rats. The effects of pyramidal tract section. Brain Res 44:313-323.

Castro AJ, Mihailoff GA (1983) Corticopontine remodelling after cortical and/or cerebellar lesions in newborn rats. J Comp Neurol 219:112-123.

Devor M, Schneider GE (1975) Neuroanatomical plasticity: the principle of conservation of total axonal arborization. In: Aspects of neural plasticity (Vital Durand F, Jeannerod M, ed), pp 191-201. Paris: INSERM 101.

Donoghue JP (1995) Plasticity of adult sensorimotor representations. Curr Opin Neurobiol 5:749-754.

Donoghue JP (1997) Limits of reorganization in cortical circuits. Cereb Cortex 7:97-99.

Fagan AM, Garber M, Barbacid M, Silos-Santiago I, Holtzman DM (1997) A role for TrkA during maturation of striatal and basal forebrain. J Neurosci 17:7644-7654.

Farmer SF, Harrison LM, Ingram DA, Stephens JA (1991) Plasticity of central motor pathways in children with hemiplegic cerebral palsy. Neurology 41:1505-1510.

Firkins SS, Bates CA, Stelzner DJ (1993) Corticospinal tract plasticity 
and astroglial reactivity after cervical spinal injury in the postnatal rat. Exp Neurol 120:1-15.

Flumerfelt BA (1980) An ultrastructural investigation of afferent connections of the red nucleus in the rat. J Anat 131:621-633.

Graybiel AM, Devor M (1974) A microelectrophoretic delivery technique for use with horseradish peroxidase. Brain Res 68:167-173.

Gwyn DG, Flumerfelt BA (1974) A comparison of the distribution of cortical and cerebellar afferents in the red nucleus of the rat. Brain Res 69:130-135.

Hepp-Reymond M-C, Wiesendanger M (1972) Unilateral pyramidotomy in monkeys: effect on force and speed of a conditioned precision grip. Brain Res 36:117-131.

Herzog A, Brösamle C (1997) "Semifree-floating" treatment: a simple and fast method to process consecutive sections for immunohistochemistry and neuronal tracing. J Neurosci Methods 72:57-63.

Jiang X, Johnson RR, Burkhalter A (1993) Visualization of dendritic morphology of cortical projection neurons by retrograde axonal tracing. J Neurosci Methods 50:45-60.

Kalil K, Reh T (1982) A light and electron microscopic study of regrowing pyramidal tract fibers. J Comp Neurol 211:265-275.

Kapfhammer JP, Schwab ME (1994) Inverse patterns of myelination and GAP-43 expression in the adult CNS: neurite growth inhibitors as regulators of neuronal plasticity? J Comp Neurol 340:194-206.

Kartje-Tillotson GL, Castro A (1980) Limb preference after unilateral pyramidotomy in adult and neonatal rats. Physiol Behav 24:293-296.

Kennard M (1936) Age and other factors in motor recovery from precentral lesions in monkeys. Am J Physiol 115:138-146.

Kennard M (1938) Reorganization of motor function in the cerebral cortex of monkeys deprived of motor and premotor areas in infancy. J Neurophysiol 1:477-496.

Kosinski RJ, Neafsey EJ, Castro AJ (1986) A comparative topographical analysis of dorsal column nuclear and cerebral cortical projections to the basilar pontine gray in rats. J Comp Neurol 244:163-173.

Kuang RZ, Kalil K (1990) Specificity of corticospinal axon arbors sprouting into denervated contralateral spinal cord. J Comp Neurol 302:461-472.

Kunkel-Bagden E, Dai, HN, Bregman BS (1993) Methods to assess the development and recovery of locomotor function after spinal cord injury in rats. Exp Neurol 119:153-164.

Leong SK, Lund RD (1973) Anomalous bilateral corticofugal pathways in albino rats after neonatal lesions. Brain Res 62:218-221.

Metz GAS, Dietz V, Schwab ME, van de Meent H (1998) The effects of unilateral pyramidal tract section on hindlimb motor performance in the rat. Behav Brain Res, in press.

Mihailoff GA, Burne RA, Woodward DJ (1978) Projections of the sensorimotor cortex to the basilar pontine nuclei in the rat: an autoradiographic study. Brain Res 145:347-354.

Murakami F, Higashi S (1988) Presence of crossed corticorubral fibers and increase of crossed projections after unilateral lesions of the cerebral cortex of the kitten: a demonstration using anterograde transport of Phaseolus vulgaris leucoagglutinin. Brain Res 447:98-108.

Nah SH, Leong SK (1976a) Bilateral corticofugal projection to the red nucleus after neonatal lesions in the albino rat. Brain Res 107:433-436.

Nah SH, Leong SK (1976b) An ultrastructural study of the anomalous corticorubral projection following neonatal lesions in the albino rat. Brain Res 111:162-166.

Nah SH, Ong LS, Leong SK (1980) Is sprouting the result of a persistent neonatal connection? Neurosci Lett 19:39-44.

Naus C, Flumerfelt BA, Hrycyshyn AW (1985a) An anterograde HRPWGA study of aberrant corticorubral projections following neonatal lesions of the rat sensorimotor cortex. Exp Brain Res 59:365-371.

Naus CG, Flumerfelt BA, Hrycyshyn AW (1985b) An HRP-TMB ultrastructural study of rubral afferents in the rat. J Comp Neurol 239:453-465.
Neafsey EJ, Bold EL, Haas G, Hurley-Gius KM, Quirk G, Sievert CF, Terreberry RR (1986) The organization of the rat motor cortex: a microstimulation mapping study. Brain Res 396:77-96.

Panto MR, Cicirata F, Angaut P, Parenti R, Serapide F (1995) The projection from the primary motor and somatic sensory cortex to the basilar pontine nuclei. A detailed electrophysiological and anatomical study in the rat. J Hirnforsch 36:7-19.

Paxinos G, Watson C (1986) The rat brain in stereotaxic coordinates, Ed 2. New York: Academic.

Reh T, Kalil K (1982) Functional role of regrowing pyramidal tract fibers. J Comp Neurol 211:276-283.

Rouiller EM, Moret V, Liang F (1993) Comparison of the connectional properties of the two forelimb areas of the rat sensorimotor cortex: support for the presence of a premotor or supplementary motor cortical area. Somatosens Mot Res 10:269-289.

Rubin BP, Spillmann AA, Bandtlow CE, Hillenbrand R, Keller F, Schwab ME (1995) Inhibition of PC12 cell attachment and neurite outgrowth by detergent solubilized CNS myelin proteins. Eur J Neurosci 7:2524-2529.

Sabel BA, Schneider GE (1988) The principle of "conservation of total axonal arborizations": massive compensatory sprouting in the hamster subcortical visual system after early tectal lesions. Exp Brain Res 73:505-518.

Schneider GE (1973) Early lesions of superior colliculus: factors affecting the formation of abnormal retinal projections. Brain Behav Evol 8:73-109.

Schnell L, Schwab ME (1990) Axonal regeneration in the rat spinal cord produced by an antibody against myelin-associated neurite growth inhibitors. Nature 343:269-272.

Schnell L, Schwab ME (1993) Sprouting and regeneration of lesioned corticospinal tract fibres in the adult rat spinal cord. Eur J Neurosci 5:1156-1171

Schnell L, Schneider R, Kolbeck R, Barde YA, Schwab ME (1994) Neurotrophin-3 enhances sprouting of corticospinal tract during development and after adult spinal cord lesion. Nature 367:170-173.

Schwab ME, Bartholdi D (1996) Degeneration and regeneration of axons in the lesioned spinal cord. Physiol Rev 76:319-370.

Schwegler G, Schwab ME, Kapfhammer JP (1995) Increased collateral sprouting of primary afferents in the myelin-free spinal cord. J Neurosci 15:2756-2767.

Thallmair M, Kapfhammer JP, Schwab ME (1997) Neutralization of myelin-associated neurite growth inhibitors increases plastic fiber growth after unilateral pyramidal tract lesions in the adult rat spinal cord. Soc Neurosci Abstr 23:609.

Thoenen H (1995) Neurotrophins and neuronal plasticity. Science 270:593-598.

Ugolini G, Kuypers HG (1986) Collaterals of corticospinal and pyramidal fibres to the pontine grey demonstrated by a new application of the fluorescent fibre labelling technique. Brain Res 365:211-227.

Vanek P, Thallmair M, Schwab ME, Kapfhammer JP (1998) Increased lesion-induced sprouting of corticospinal fibers in the myelin-free rat spinal cord. Eur J Neurosci 10:45-56.

Whishaw IQ, Kolb B (1988) Sparing of skilled forelimb reaching and corticospinal projections after neonatal motor cortex removal or hemidecortication in the rat: support for the Kennard doctrine. Brain Res 451:97-114.

Whishaw IQ, Pellis SM, Gorny B, Kolb B, Tetzlaff W (1993) Proximal and distal impairments in rat forelimb use in reaching follow unilateral pyramidal tract lesions. Behav Brain Res 56:59-76.

Wiesendanger R, Wiesendanger M (1982) The corticopontine system in the rat. II. The projection pattern. J Comp Neurol 208:227-238. 